CUBO A Mathematical Journal Vol.13, № 02, (1-35). June 2011

\title{
Homogeneous Besov Spaces associated with the spherical mean operator
}

\author{
L.T.RACHDI AND A.RouZ \\ Department of Mathematics, \\ Faculty of Sciences of Tunis, \\ 2092 El Manar 2 Tunis, Tunisia. \\ email: ahlemrouz@yahoo.fr
}

\begin{abstract}
We define and study homogeneous Besov spaces associated with the spherical mean operator. We establish some results of completeness, continuous embeddings and density of subspaces. Next, we define a discrete equivalent norm on this space and we give other properties.
\end{abstract}

\section{RESUMEN}

Definimos y estudiamos los espacios homogneos Besov asociados con el operador esférico medio. Se establecen algunos resultados de la exhaustividad, de inclusiones continuas y de la densidad de subespacios. A continuación, se define una norma equivalente discreta en este espacio y se dan otras propiedades.

Keywords and phrases:: Spherical mean operator, Besov space, Banach space, Fourier transform.

Mathematics Subject Classification: 46E35, 44A35. 


\section{Introduction}

For a continuous function $f$ on $\mathbb{R} \times \mathbb{R}^{n}$, even with respect to the first variable, the spherical mean operator $\mathscr{R}$ is defined as

$$
\mathscr{R}(f)(r, x)=\int_{S^{n}} f(r \eta, x+r \xi) d \sigma_{n}(\eta, \xi) ; \quad(r, x) \in \mathbb{R} \times \mathbb{R}^{n},
$$

where $S^{n}$ is the unit sphere, i.e. $S^{n}=\left\{(\eta, \xi) \in \mathbb{R} \times \mathbb{R}^{n} ; \eta^{2}+|\xi|^{2}=1\right\}$ and $\sigma_{n}$ is the surface measure on $S^{n}$ normalized to have total measure one.

The dual of the spherical mean operator ${ }^{\mathrm{t}} \mathscr{R}$ is defined by

$$
\mathrm{t} \mathscr{R}(\mathrm{g})(\mathrm{r}, \mathrm{x})=\frac{\Gamma\left(\frac{\mathrm{n}+1}{2}\right)}{\pi^{\frac{n+1}{2}}} \int_{\mathbb{R}^{n}} \mathrm{~g}\left(\sqrt{r^{2}+|x-y|^{2}}, y\right) d y,
$$

where $d y$ is the Lebesgue measure on $\mathbb{R}^{n}$.

The spherical mean operator $\mathscr{R}$ and its dual ${ }^{\mathrm{t}} \mathscr{R}$ play an important role and have many applications, for example, in image processing of so-called synthetic aperture radar (SAR) data [14, 15, or in the linearized inverse scattering problem in acoustics 9 .

Many aspects of such operator have been studied [1, 3, 6, 18, 21]. In particular, in [18] the first author with the others associated to the spherical mean operator the Fourier transform defined by

$$
\forall(\mu, \lambda) \in \Gamma, \quad \mathscr{F}(f)(\mu, \lambda)=\int_{\mathbb{R}^{n}} \int_{0}^{+\infty} f(r, x) \varphi_{\mu, \lambda}(r, x) d v_{n}(r, x),
$$

where

- $\varphi_{\mu, \lambda}$ is the function defined by

$$
\forall(r, x) \in \mathbb{R} \times \mathbb{R}^{n}, \varphi_{\mu, \lambda}(r, x)=\mathscr{R}\left(\cos (\mu .) e^{-i\langle\lambda \mid \cdot\rangle}\right)(r, x) .
$$

- $v_{\mathrm{n}}$ is the measure defined on $\left[0,+\infty\left[\times \mathbb{R}^{n}\right.\right.$, by

$$
d v_{n}(r, x)=\frac{1}{2^{\frac{n-1}{2}} \Gamma\left(\frac{n+1}{2}\right)(2 \pi)^{\frac{n}{2}}} r^{n} d r \otimes d x
$$

- $\Gamma$ is the set given by

$$
\Gamma=\mathbb{R} \times \mathbb{R}^{n} \cup\left\{(i \mu, \lambda) ;(\mu, \lambda) \in \mathbb{R} \times \mathbb{R}^{n},|\mu| \leqslant|\lambda|\right\} .
$$

They have constructed the harmonic analysis related to the Fourier transform $\mathscr{F}$ (Inversion formula, Schwartz theorem, Paley-Wiener theorem, Plancherel theorem).

There are many ways to define Besov Spaces [4, 5, 13, 16, 20, 23. It is well known that Besov spaces can be defined for instance in terms of convolutions $f * \phi_{t}$ with different kinds of smooth functions $\phi$ and that can be also described by means of differences $\triangle_{x} f[10,11,22$. 
In this work, we define and study a class of homogeneous Besov spaces connected with the spherical mean operator $\mathscr{R}$. More precisely, let $\phi$ be a smooth function on $\mathbb{R} \times \mathbb{R}^{n}$, even with respect to the first variable. For all $p, q \in[1,+\infty]$ and $\gamma \in \mathbb{R}$, we define the Besov space $\mathscr{B} p, q, q([0,+\infty[\times$ $\mathbb{R}^{n}$ ) to be the space of tempered distributions $f$ on $\mathbb{R} \times \mathbb{R}^{n}$, even with respect to the first variable such that

$$
\mathrm{f}=\int_{0}^{+\infty} \mathrm{f} * \phi_{\mathrm{t}} * \phi_{\mathrm{t}} \frac{\mathrm{dt}}{\mathrm{t}}
$$

where $*$ is the convolution product associated with the spherical mean operator and $\phi_{\mathrm{t}} ; \mathrm{t}>0$ is the dilated function of $\phi$ defined by

$$
\forall(r, x) \in\left[0,+\infty\left[\times \mathbb{R}^{n}, \quad \phi_{t}(r, x)=\frac{1}{t^{2 n+1}} \phi\left(\frac{r}{t}, \frac{x}{t}\right)\right.\right.
$$

(see Definition 10 below).

The space $\mathscr{B}_{\mathrm{p}, \mathrm{q}}^{\gamma, \phi}\left(\left[0,+\infty\left[\times \mathbb{R}^{\mathrm{n}}\right)\right.\right.$ is equipped firstly with the norm

$$
M_{p, q}^{\gamma, \phi}(f)= \begin{cases}\left(\int_{0}^{+\infty}\left(\frac{\left\|f * \phi_{t}\right\|_{p, v_{n}}}{t^{\gamma}}\right)^{q} \frac{d t}{t}\right)^{\frac{1}{q}}, & \text { if } 1 \leqslant q<+\infty \\ \operatorname{esssup}_{t>0} \frac{\left\|f * \phi_{t}\right\|_{p, v_{n}}}{t^{\gamma}}, & \text { if } q=+\infty\end{cases}
$$

with

$$
\left\|f * \phi_{\mathrm{t}}\right\|_{p, v_{n}}= \begin{cases}\left(\int_{\mathbb{R}^{n}} \int_{0}^{+\infty}\left|\mathrm{f} * \phi_{\mathrm{t}}(\mathrm{r}, \mathrm{x})\right|^{\mathrm{p}} \mathrm{d} v_{\mathrm{n}}(\mathrm{r}, \mathrm{x})\right)^{\frac{1}{\mathrm{p}}}, & \text { if } p \in[1,+\infty[; \\ \operatorname{esssup}_{(\mathrm{r}, \mathrm{x}) \in\left[0,+\infty\left[\times \mathbb{R}^{n}\right.\right.}\left|\mathrm{f} * \phi_{\mathrm{t}}(\mathrm{r}, \mathrm{x})\right|, & \text { if } \mathrm{p}=+\infty .\end{cases}
$$


Then we have established the coming results

- The Besov space $\mathscr{B}_{\mathrm{p}, \mathrm{q}}^{\gamma, \phi}\left(\left[0,+\infty\left[\times \mathbb{R}^{\mathrm{n}}\right)\right.\right.$ is independent of the choice of the function $\phi$ and will be denoted by $\mathscr{B}_{p, q}^{\gamma}\left(\left[0,+\infty\left[\times \mathbb{R}^{n}\right)\right.\right.$. This means that for all smooth functions $\phi$ and $\psi$, there exists a positive constant $C_{\phi, \psi}$ such that

$$
\forall f \in \mathscr{B}_{\mathfrak{p}, \mathbf{q}}^{\gamma, \phi}\left(\left[0,+\infty\left[\times \mathbb{R}^{n}\right), M_{\mathfrak{p}, \mathbf{q}}^{\gamma, \phi}(f) \leqslant C_{\phi, \psi} M_{\mathfrak{p}, \mathbf{q}}^{\gamma, \psi}(f) .\right.\right.
$$

- The space $\mathscr{B}_{\mathrm{p}, \mathrm{q}}^{\gamma}\left(\left[0,+\infty\left[\times \mathbb{R}^{\mathrm{n}}\right)\right.\right.$ is homogeneous with degree equal to $(2 n+1) / p-\gamma-2 n-1$, that is for all $f \in \mathscr{B}_{p, q}^{\gamma}\left(\left[0,+\infty\left[\times \mathbb{R}^{n}\right)\right.\right.$ and $t>0$, the distribution $f_{\mathrm{t}}$ belongs to the space $\mathscr{B}_{\mathrm{p}, \mathrm{q}}^{\gamma}\left(\left[0,+\infty\left[\times \mathbb{R}^{\mathrm{n}}\right)\right.\right.$ and we have

$$
M_{p, q}^{\gamma, \phi}\left(f_{t}\right)=t^{\frac{2 n+1}{p}-\gamma-2 n-1} M_{p, q}^{\gamma, \phi}(f) .
$$

- The Besov space is a Banach one when $\gamma<(2 n+1) / p$.

We have also proved some continuous embeddings and density of subspaces.

Next, we define the following discrete norm on the space $\mathscr{B}_{\mathrm{p}, \mathrm{q}}^{\gamma}\left(\left[0,+\infty\left[\times \mathbb{R}^{\mathrm{n}}\right)\right.\right.$ by setting

$$
N_{p, q}^{\gamma, \phi}(f)= \begin{cases}\left(\sum_{k \in \mathbb{Z}}\left(\frac{\left\|f * \phi_{2^{k}}\right\|_{p, v_{n}}}{2^{k \gamma}}\right)^{q}\right)^{\frac{1}{q}}, & \text { if } 1 \leqslant q<+\infty ; \\ \operatorname{esssup}_{k \in \mathbb{Z}} \frac{\left\|f * \phi_{2^{k}}\right\|_{p, v_{n}}}{2^{k \gamma}}, & \text { if } q=+\infty .\end{cases}
$$

We show that this norm defines the same topology as the norm $M_{\mathfrak{p}, \mathbf{q}}^{\gamma}$. We prove that this space is homogeneous in a weaker sense when equipped with the norm $\mathrm{N}_{\mathbf{p}, \mathbf{q}}^{\gamma, \phi}$, that is there exist two positive constants $C_{1}$ and $C_{2}$ such that for all $f \in \mathscr{B}_{p, q}^{\gamma}\left(\left[0,+\infty\left[\times \mathbb{R}^{n}\right)\right.\right.$ and $t>0$

$$
C_{1} t^{\frac{2 n+1}{p}-2 n-1-\gamma} N_{p, q}^{\gamma, \phi}(f) \leqslant N_{p, q}^{\gamma, \phi}\left(f_{t}\right) \leqslant C_{2} t^{\frac{2 n+1}{p}-2 n-1-\gamma} N_{p, q}^{\gamma, \phi}(f) .
$$

Finally, we establish some new continuous embedding.

\section{Fourier transform associated with the spherical mean op- erator}

In this section, we recall some harmonic analysis results related to the Fourier transform associated with the spherical mean operator.

Let $\varphi_{\mu, \lambda},(\mu, \lambda) \in \mathbb{C} \times \mathbb{C}^{n}$, be the function defined by

$$
\forall(r, x) \in \mathbb{R} \times \mathbb{R}^{n}, \varphi_{\mu, \lambda}(r, x)=\mathscr{R}\left(\cos (\mu .) e^{-i\langle\lambda \mid \cdot\rangle}\right)(r, x) .
$$

It's well known $([18,21])$ that 
i. The function $\varphi_{\mu, \lambda}$ is given by

$$
\forall(r, x) \in \mathbb{R} \times \mathbb{R}^{n}, \quad \varphi_{\mu, \lambda}(r, x) e^{-i\langle\lambda \mid x\rangle} j_{(n-1) / 2}\left(r \sqrt{\mu^{2}+\lambda_{1}^{2}+\ldots+\lambda_{n}^{2}}\right),
$$

where $j_{(n-1) / 2}$ is the modified Bessel function defined by

$$
j_{\frac{n-1}{2}}(s)=2^{\frac{n-1}{2}} \Gamma\left(\frac{n+1}{2}\right) \frac{J_{\frac{n-1}{2}}(s)}{s^{\frac{n-1}{2}}}=\Gamma\left(\frac{n+1}{2}\right) \sum_{k=0}^{+\infty} \frac{(-1)^{k}}{k ! \Gamma(\alpha+k+1)}\left(\frac{s}{2}\right)^{2 k},
$$

and $J_{(n-1) / 2}$ is the Bessel function of first kind and index $(n-1) / 2$ [7, 8, 17, 27].

ii. For all $(\mu, \lambda) \in \mathbb{C} \times \mathbb{C}^{n}, \varphi_{\mu, \lambda}$ is the unique infinitely differentiable function on $\mathbb{R} \times \mathbb{R}^{n}$, even with respect to the first variable, satisfying

$$
\left\{\begin{array}{l}
D_{j} u\left(r, x_{1}, \ldots, x_{n}\right)=-i \lambda_{j} u\left(r, x_{1}, \ldots, x_{n}\right), \quad 1 \leqslant j \leqslant n, \\
\Xi u\left(r, x_{1}, \ldots, x_{n}\right)=-\mu^{2} u\left(r, x_{1}, \ldots, x_{n}\right), \\
u(0, \ldots, 0)=1, \frac{\partial u}{\partial r}\left(0, x_{1}, \ldots, x_{n}\right)=0, \quad \forall\left(x_{1}, \ldots, x_{n}\right) \in \mathbb{R}^{n} .
\end{array}\right.
$$

where

$$
D_{j}=\frac{\partial}{\partial x_{j}} ; \quad 1 \leqslant j \leqslant n, \quad \text { and } \quad \Xi=\frac{\partial^{2}}{\partial r^{2}}+\frac{n}{r} \frac{\partial}{\partial r}-\sum_{j=1}^{n} D_{j}^{2} .
$$

iii. The function $\varphi_{\mu, \lambda}$ is bounded on $\mathbb{R} \times \mathbb{R}^{n}$ if, and only if $(\mu, \lambda)$ belongs to the set $\Gamma$ given by

$$
\Gamma=\mathbb{R} \times \mathbb{R}^{n} \cup\left\{(i \mu, \lambda) ;(\mu, \lambda) \in \mathbb{R} \times \mathbb{R}^{n},|\mu| \leqslant|\lambda|\right\} .
$$

In this case, we have

$$
\sup _{(r, x) \in \mathbb{R} \times \mathbb{R}^{n}}\left|\varphi_{\mu, \lambda}(r, x)\right|=1
$$

We denote by

- $L^{p}\left(d v_{n}\right), p \in[1,+\infty]$, the space of measurable functions $f$ on $\left[0,+\infty\left[\times \mathbb{R}^{n}\right.\right.$, such that

$$
\|f\|_{p, v_{n}}= \begin{cases}\left(\int_{\mathbb{R}^{n}} \int_{0}^{+\infty}|f(r, x)|^{p} d v_{n}(r, x)\right)^{\frac{1}{p}}<+\infty, & \text { if } p \in[1,+\infty[; \\ \operatorname{esssup}_{(r, x) \in\left[0,+\infty\left[\times \mathbb{R}^{n}\right.\right.}|f(r, x)|<+\infty, & \text { if } p=+\infty,\end{cases}
$$

where $v_{n}$ is the measure defined in the introduction.

- $\Gamma_{+}$the subset of $\Gamma$ given by

$$
\Gamma_{+}=\left[0,+\infty\left[\times \mathbb{R}^{n} \cup\left\{(i \mu, \lambda) ;(\mu, \lambda) \in \mathbb{R} \times \mathbb{R}^{n}, 0 \leqslant \mu \leqslant|\lambda|\right\} .\right.\right.
$$


- $\mathcal{B}_{\Gamma_{+}}$the $\sigma$-algebra on $\Gamma_{+}$defined by

$$
\mathcal{B}_{\Gamma_{+}}=\theta^{-1}\left(\mathcal{B}_{\left[0,+\infty\left[\times \mathbb{R}^{n}\right.\right.}\right),
$$

where $\theta$ is the bijective function defined on $\Gamma_{+}$by

$$
\theta(\mu, \lambda)=\left(\sqrt{\mu^{2}+\lambda^{2}}, \lambda\right) .
$$

- $\gamma_{\mathrm{n}}$ the measure defined on $\Gamma_{+}$by

$$
\gamma_{\mathfrak{n}}(A)=\nu_{\mathfrak{n}}(\theta(A)) ; \quad A \in \mathcal{B}_{\Gamma_{+}} .
$$

- $L^{p}\left(d \gamma_{n}\right), p \in[1,+\infty]$, the space of measurable functions on $\Gamma_{+}$satisfying

$$
\|f\|_{p, \gamma_{n}}<+\infty .
$$

Then we have the coming properties

Proposition 1. i) For all non negative measurable function $f$ on $\Gamma_{+}$(respectively integrable on $\Gamma_{+}$ with respect to the measure $d \gamma_{n}$ ), we have

$$
\begin{gathered}
\iint_{\Gamma_{+}} f(\mu, \lambda) d \gamma_{n}(\mu, \lambda) \frac{1}{2^{\frac{n-1}{2}} \Gamma\left(\frac{n+1}{2}\right)(2 \pi)^{\frac{n}{2}}}\left\{\int_{\mathbb{R}^{n}} \int_{0}^{+\infty} f(\mu, \lambda)\left(\mu^{2}+|\lambda|^{2}\right)^{\frac{n-1}{2}} \mu d \mu d \lambda\right. \\
\left.+\int_{\mathbb{R}^{n}} \int_{0}^{|\lambda|} f(i \mu, \lambda)\left(|\lambda|^{2}-\mu^{2}\right)^{\frac{n-1}{2}} \mu d \mu d \lambda\right\} .
\end{gathered}
$$

ii) For all non negative measurable function $g$ on $\left[0,+\infty\left[\times \mathbb{R}^{n}\right.\right.$ (respectively integrable on $[0,+\infty[\times$ $\mathbb{R}^{n}$ with respect to the measure $d v_{n}$ ), the function $g \circ \theta$ is measurable positive on $\Gamma_{+}$(respectively integrable on $\Gamma_{+}$with respect to the measure $\mathrm{d} \gamma_{n}$ ) and we have

$$
\int_{\mathbb{R}^{n}} \int_{0}^{\infty} g(r, x) d v_{n}(r, x)=\iint_{\Gamma_{+}} g \circ \theta(\mu, \lambda) d \gamma_{n}(\mu, \lambda) .
$$

In the following, we shall define the translation operator and the convolution product associated with the spherical mean operator. For this, we use the product formula for the function $\varphi_{\mu, \lambda}$, for all $(r, x),(s, y) \in \mathbb{R} \times \mathbb{R}^{n}$, we have

$$
\varphi_{\mu, \lambda}(r, x) \varphi_{\mu, \lambda}(s, y) \frac{\Gamma\left(\frac{n+1}{2}\right)}{\sqrt{\pi} \Gamma\left(\frac{n}{2}\right)} \int_{0}^{\pi} \varphi_{\mu, \lambda}\left(\sqrt{r^{2}+s^{2}+2 r s \cos \theta}, x+y\right) \sin ^{n-1}(\theta) d \theta
$$

Definition 2. i) For all $(r, x) \in\left[0,+\infty\left[\times \mathbb{R}^{n}\right.\right.$, the translation operator $\tau_{(r, x)}$ associated with the spherical mean operator is defined on $L^{p}\left(d v_{n}\right), p \in[1,+\infty]$, by

$$
\tau_{(r, x)}(f)(s, y)=\frac{\Gamma\left(\frac{n+1}{2}\right)}{\sqrt{\pi} \Gamma\left(\frac{n}{2}\right)} \int_{0}^{\pi} f\left(\sqrt{r^{2}+s^{2}+2 r s \cos \theta}, x+y\right) \sin ^{n-1}(\theta) d \theta .
$$


ii) The convolution product of $f, g \in L^{1}\left(d v_{n}\right)$ is defined by

$$
\forall(r, x) \in\left[0,+\infty\left[\times \mathbb{R}^{n}, \quad f * g(r, x)=\int_{\mathbb{R}^{n}} \int_{0}^{+\infty} f(s, y) \tau_{(r,-x)}(\check{g})(s, y) d v_{n}(s, y),\right.\right.
$$

where

$$
\check{g}(s, y)=g(s,-y) .
$$

We have the following properties

- For all $(r, x),(s, y) \in\left[0,+\infty\left[\times \mathbb{R}^{n}\right.\right.$, the relation (2.4) can be written

$$
\tau_{(r, x)}\left(\varphi_{\mu, \lambda}\right)(s, y) \varphi_{\mu, \lambda}(r, x) \varphi_{\mu, \lambda}(s, y)
$$

- If $f \in L^{p}\left(d v_{n}\right), 1 \leqslant p \leqslant+\infty$, then for all $(s, y) \in\left[0,+\infty\left[\times \mathbb{R}^{n}\right.\right.$, the function $\tau_{(s, y)}(f)$ belongs to $L^{p}\left(d v_{n}\right)$ and we have

$$
\left\|\tau_{(s, y)}(f)\right\|_{p, v_{n}} \leqslant\|f\|_{p, v_{n}} .
$$

- Let $p, q, r \in[1,+\infty]$ such that $\frac{1}{r}=\frac{1}{p}+\frac{1}{q}-1$. Then for all $f \in L^{p}\left(d v_{n}\right)$ and $g \in L^{q}\left(d v_{n}\right)$, the function $f * g$ belongs to $L^{r}\left(d v_{n}\right)$ and we have

$$
\|f * g\|_{r, v_{n}} \leqslant\|f\|_{p, v_{n}}\|g\|_{q, v_{n}} .
$$

Now, we will define the Fourier transform $\mathscr{F}$ connected with the spherical mean operator and we recall some properties that we need in the next section.

Definition 3. The Fourier transform associated with the spherical mean operator is defined on $\mathrm{L}^{1}\left(\mathrm{~d} v_{\mathrm{n}}\right)$ by

$$
\forall(\mu, \lambda) \in \Gamma, \quad \mathscr{F}(f)(\mu, \lambda)=\int_{\mathbb{R}^{n}} \int_{0}^{+\infty} f(r, x) \varphi_{\mu, \lambda}(r, x) d v_{n}(r, x),
$$

where $\Gamma$ is the set defined by the relation (2.2).

The Fourier transform $\mathscr{F}$ satisfies the properties

- For every $f$ in $L^{1}\left(d v_{n}\right)$ and $(r, x) \in\left[0,+\infty\left[\times \mathbb{R}^{n}\right.\right.$, we have

$$
\forall(\mu, \lambda) \in \Gamma, \quad \mathscr{F}\left(\tau_{(r,-x)}(f)\right)(\mu, \lambda) \varphi_{\mu, \lambda}(r, x) \mathscr{F}(f)(\mu, \lambda) .
$$

- For all $f, g \in L^{1}\left(d v_{n}\right)$, we have

$$
\forall(\mu, \lambda) \in \Gamma, \quad \mathscr{F}(f * g)(\mu, \lambda)=\mathscr{F}(f)(\mu, \lambda) \mathscr{F}(g)(\mu, \lambda) .
$$

- For all $f \in L^{1}\left(d v_{n}\right)$, we have

$$
\forall(\mu, \lambda) \in \Gamma, \quad \mathscr{F}(f)(\mu, \lambda) \widetilde{\mathscr{F}}(f) \circ \theta(\mu, \lambda),
$$


where

$$
\forall(\mu, \lambda) \in \mathbb{R} \times \mathbb{R}^{n}, \quad \widetilde{\mathscr{F}}(f)(\mu, \lambda)=\int_{\mathbb{R}^{n}} \int_{0}^{+\infty} f(r, x) j_{\frac{n-1}{2}}(r \mu) e^{-i\langle\lambda \mid x\rangle} d v_{n}(r, x)
$$

and $\theta$ is the function defined by the relation (2.3).

Theorem 4. (Inversion formula for $\mathscr{F})$ Let $f \in \mathrm{L}^{1}\left(\mathrm{~d} v_{\mathrm{n}}\right)$ such that the function $\mathscr{F}(\mathrm{f})$ belongs to $\mathrm{L}^{1}\left(\mathrm{~d} \gamma_{n}\right)$, then for almost every $(r, x) \in\left[0,+\infty\left[\times \mathbb{R}^{n}\right.\right.$, we have

$$
f(r, x)=\iint_{\Gamma_{+}} \mathscr{F}(f)(\mu, \lambda) \overline{\varphi_{\mu, \lambda}(r, x)} \mathrm{d} \gamma_{\mathfrak{n}}(\mu, \lambda) .
$$

We denote by

- $\mathscr{E}_{*}\left(\mathbb{R} \times \mathbb{R}^{n}\right)$ the space of infinitely differentiable functions on $\mathbb{R} \times \mathbb{R}^{n}$, even with respect to the first variable.

- $S_{*}\left(\mathbb{R} \times \mathbb{R}^{n}\right)$ the subspace of $\mathscr{E}_{*}\left(\mathbb{R} \times \mathbb{R}^{n}\right)$ consisting of functions rapidly decreasing together with all their derivatives.

- $S_{*}(\Gamma)$ the space of functions $f: \Gamma \longrightarrow \mathbb{C}$ infinitely differentiable, even with respect to the first variable and rapidly decreasing together with all their derivatives, i.e

$$
\forall k_{1}, k_{2} \in \mathbb{N}, \forall \alpha \in \mathbb{N}^{n}, \quad \sup _{(\mu, \lambda) \in \Gamma}\left(1+\mu^{2}+2|\lambda|^{2}\right)^{k_{1}}\left|\left(\frac{\partial}{\partial \mu}\right)^{k_{2}} D_{\lambda}^{\alpha} f(\mu, \lambda)\right|<+\infty,
$$

where

$$
\frac{\partial f}{\partial \mu}(\mu, \lambda) \begin{cases}\frac{\partial}{\partial r}(f(r, \lambda)), & \text { if } \mu=r \in \mathbb{R} \\ \frac{1}{i} \frac{\partial}{\partial t}(f(i t, \lambda)), & \text { if } \mu=i t,|t| \leqslant|\lambda|\end{cases}
$$

and

$$
\mathrm{D}_{\lambda}^{\alpha}=\left(\frac{\partial}{\partial \lambda_{1}}\right)^{\alpha_{1}} \ldots\left(\frac{\partial}{\partial \lambda_{n}}\right)^{\alpha_{n}} .
$$

- $S_{*}^{\prime}\left(\mathbb{R} \times \mathbb{R}^{n}\right)$ and $S_{*}^{\prime}(\Gamma)$ are respectively the topological dual spaces of $S_{*}\left(\mathbb{R} \times \mathbb{R}^{n}\right)$ and $S_{*}(\Gamma)$.

Each of these spaces is equipped with its usual topology.

Theorem 5. (Schwartz theorem) 2, 18, i) The Fourier transform $\mathscr{F}$ is a topological isomorphism from $S_{*}\left(\mathbb{R} \times \mathbb{R}^{n}\right)$ onto $S_{*}(\Gamma)$. The inverse mapping is given by

$$
\forall(r, x) \in \mathbb{R} \times \mathbb{R}^{n}, \mathscr{F}^{-1}(\mathrm{f})(\mathrm{r}, \mathrm{x})=\iint_{\Gamma_{+}} \mathrm{f}(\mu, \lambda) \overline{\varphi_{\mu, \lambda}(\mathrm{r}, \mathrm{x})} \mathrm{d} \gamma_{\mathrm{n}}(\mu, \lambda) .
$$

ii) (Plancherel formula) For all $f, g \in S_{*}\left(\mathbb{R} \times \mathbb{R}^{n}\right)$, we have

$$
\int_{0}^{+\infty} \int_{\mathbb{R}^{n}} f(r, x) \overline{g(r, x)} d v_{n}(r, x)=\iint_{\Gamma_{+}} \mathscr{F}(f)(\mu, \lambda) \overline{\mathscr{F}(g)(\mu, \lambda)} d \gamma_{n}(\mu, \lambda) .
$$


In particular

$$
\|\mathscr{F}(\mathrm{f})\|_{2, \gamma_{n}}\|\mathrm{f}\|_{2, v_{n}} .
$$

Theorem 6. (Plancherel theorem) The Fourier transform $\mathscr{F}$ can be extended to an isometric isomorphism from $L^{2}\left(d v_{n}\right)$ onto $L^{2}\left(d \gamma_{n}\right)$.

For $\mathrm{T} \in \mathrm{S}_{*}^{\prime}\left(\mathbb{R} \times \mathbb{R}^{n}\right)$, we put

$$
\langle\mathscr{F}(\mathrm{T}), \varphi\rangle=\left\langle\mathrm{T}, \mathscr{F}^{-1}(\varphi)\right\rangle ; \quad \varphi \in \mathrm{S}_{*}(\Gamma) .
$$

Then from Theorem 5, we get the following result

Corollary 7. The transform $\mathscr{F}$ defined by the relation (2.13) is a topological isomorphism from $S_{*}^{\prime}\left(\mathbb{R} \times \mathbb{R}^{n}\right)$ onto $S_{*}^{\prime}(\Gamma)$.

Proposition 8 . i) Let $f \in \mathscr{E}_{*}\left(\mathbb{R} \times \mathbb{R}^{\mathrm{n}}\right)$, $f$ slowly increasing and let $g \in S_{*}\left(\mathbb{R} \times \mathbb{R}^{n}\right)$. Then the function $\mathrm{f} * \mathrm{~g}$ belongs to the space $\mathscr{E}_{*}\left(\mathbb{R} \times \mathbb{R}^{\mathrm{n}}\right)$.

ii) For all $f \in S_{*}\left(\mathbb{R} \times \mathbb{R}^{n}\right)$ and $T \in S_{*}^{\prime}\left(\mathbb{R} \times \mathbb{R}^{n}\right)$. The function $T * f$ defined by

$$
\forall(r, x) \in \mathbb{R} \times \mathbb{R}^{n}, \quad \mathrm{~T} * \mathrm{f}(\mathrm{r}, \mathrm{x})=\left\langle\mathrm{T}, \tau_{(\mathrm{r},-x)}(\check{\mathrm{f}})\right\rangle
$$

belongs to the space $\mathscr{E}_{*}\left(\mathbb{R} \times \mathbb{R}^{n}\right)$ and is slowly increasing. Moreover, we have

$$
\mathscr{F}\left(\mathrm{T}_{\mathrm{T} * \mathrm{f}}\right)=\mathscr{F}(\check{\mathrm{f}}) \mathscr{F}(\mathrm{T})
$$

\section{Besov spaces}

This section contains the main result of this paper. Indeed, we define and study a class of Besov spaces $\mathscr{B}_{\mathrm{p}, \mathrm{q}}^{\gamma}\left(\left[0,+\infty\left[\times \mathbb{R}^{n}\right)\right.\right.$, where $\phi$ is a smooth function. We show that this space is independant of the choice of $\phi$ and is a Banach space for $\gamma<(2 n+1) / p$. Next, we prove that $\mathscr{B}_{\mathbf{p}, \mathbf{q}}^{\gamma, \phi}([0,+\infty[\times$ $\left.\mathbb{R}^{n}\right)$ is an homogeneous space with degree equal to $(2 n+1) / p-\gamma-2 n-1$.

Lemma 9. Let $\mathrm{a}, \mathrm{b}, \mathrm{a}_{1}, \mathrm{~b}_{1}$ be real numbers such that $0<\mathrm{a}_{1}<\mathrm{a}<\mathrm{b}<\mathrm{b}_{1}$. Then there exists a function $\psi \in S_{*}\left(\mathbb{R} \times \mathbb{R}^{n}\right)$ satisfying the following assumptions

i) $\forall(\mu, \lambda) \in \Gamma, \quad \mathscr{F}(\psi)(\mu, \lambda) \geqslant 0$.

ii) $\forall(\mu, \lambda) \in \Gamma ; \quad a^{2} \leqslant \mu^{2}+2|\lambda|^{2} \leqslant b^{2}, \quad \mathscr{F}(\psi)(\mu, \lambda)=C$ where $\mathrm{C}$ is a positive constante.

iii) $\mathscr{F}(\psi)(\mu, \lambda)=0$ if $\mu^{2}+2|\lambda|^{2}>b_{1}^{2}$ or $\mu^{2}+2|\lambda|^{2}<a_{1}^{2}$. 
iv) For all $(\mu, \lambda) \in \Gamma \backslash\{(0,0)\}$,

$$
\int_{0}^{+\infty}(\mathscr{F}(\psi)(t \mu, t \lambda))^{2} \frac{d t}{t}=1
$$

Proof. From Uryshon's lemma, there exists an infinitely differentiable function $\omega$ on $\mathbb{R}$ such that

- $\forall \mathrm{t} \in \mathbb{R} ; \quad 0 \leqslant \omega(\mathrm{t}) \leqslant 1$.

- $\forall \mathrm{t} \in[\mathrm{a}, \mathrm{b}] ; \quad \omega(\mathrm{t})=1$.

- $\operatorname{supp}(\omega) \subset] a_{1}, b_{1}[$.

Let $\mathrm{g}$ be the function defined on $\mathbb{R} \times \mathbb{R}^{n}$ by

$$
g(r, x)=\frac{\omega\left(\sqrt{r^{2}+|x|^{2}}\right)}{\left(\int_{0}^{+\infty}(\omega(t))^{2} \frac{d t}{t}\right)^{\frac{1}{2}}},
$$

then the function $\mathrm{g}$ belongs to the space $S_{*}\left(\mathbb{R} \times \mathbb{R}^{n}\right)$. Since, the transform $\widetilde{\mathscr{F}}$ defined by the relation (2.11) is a topological isomorphism from the space $S_{*}\left(\mathbb{R} \times \mathbb{R}^{n}\right)$ onto itself [24, 25], then there exists $\psi \in S_{*}\left(\mathbb{R} \times \mathbb{R}^{n}\right)$ such that $\widetilde{\mathscr{F}}(\psi)=\mathrm{g}$. Thus, by the relation (2.10), we deduce that the function $\psi$ satisfies the hypothesis of the lemma.

\section{We denote by}

- $\mathscr{D}_{*}(\Gamma)$ the space of real infinitely differentiable functions $\mathrm{g}$ on $\Gamma$, even with respect to the first variable such that, there exist two positive real numbers

$0<a<b$ verifying

$$
g(\mu, \lambda)=0 \quad \text { if } \mu^{2}+2|\lambda|^{2}<a^{2} \text { or } \mu^{2}+2|\lambda|^{2}>b^{2} .
$$

- $S_{*, 0}\left(\mathbb{R} \times \mathbb{R}^{n}\right)$ the subspace of $S_{*}\left(\mathbb{R} \times \mathbb{R}^{n}\right)$ consisting of functions $f$ such that $\mathscr{F}(f)$ belongs to the space $\mathscr{D}_{*}(\Gamma)$.

- $S_{*, 0}^{1}\left(\mathbb{R} \times \mathbb{R}^{n}\right)$ the subspace of $S_{*, 0}\left(\mathbb{R} \times \mathbb{R}^{n}\right)$ formed by the functions $f$ such that

$$
\forall(\mu, \lambda) \in \Gamma \backslash\{(0,0)\}, \quad \int_{0}^{+\infty}(\mathscr{F}(\mathrm{f})(\mathrm{t} \mu, \mathrm{t} \lambda))^{2} \frac{\mathrm{dt}}{\mathrm{t}}=1 .
$$

These functions are known as wavelets on $\left[0,+\infty\left[\times \mathbb{R}^{n}[19,26]\right.\right.$. 
- $\mathrm{L}^{\mathrm{p}}\left(\frac{\mathrm{dt}}{\mathrm{t}}\right) ; p \in[1,+\infty]$, the space of measurable functions on $] 0,+\infty[$ such that

$$
\|f\|_{L^{p}\left(\frac{d t}{t}\right)}= \begin{cases}\left(\int_{0}^{+\infty}|f(t)|^{p} \frac{d t}{t}\right)^{\frac{1}{p}}<+\infty, & 1 \leqslant p<+\infty ; \\ \underset{t>0}{\operatorname{esssup}}|f(t)|<+\infty, & p=+\infty .\end{cases}
$$

- $\star$ the convolution product defined on the group (] $0,+\infty[,$.$) by$

$$
f \star g(s)=\int_{0}^{+\infty} f(t) g\left(\frac{s}{t}\right) \frac{d t}{t} .
$$

- For all measurable function $\phi$ on $\left[0,+\infty\left[\times \mathbb{R}^{n}\right.\right.$, the dilated $\phi_{t} ; t>0$ of $\phi$ is defined by

$$
\forall(r, x) \in\left[0,+\infty\left[\times \mathbb{R}^{n}, \quad \phi_{t}(r, x)=\frac{1}{t^{2 n+1}} \phi\left(\frac{r}{t}, \frac{x}{t}\right) .\right.\right.
$$

Then we have the following properties

- Let $p, q, r \in[1,+\infty]$ such that $\frac{1}{p}+\frac{1}{q}=1+\frac{1}{r}$. Then for all $f \in L^{p}\left(\frac{d t}{t}\right)$ and $g \in L^{q}\left(\frac{d t}{t}\right)$, the function $f \star g$ belongs to $L^{r}\left(\frac{d t}{t}\right)$ and we have

$$
\|f \star g\|_{L^{r}\left(\frac{d t}{t}\right)} \leqslant\|f\|_{L^{p}\left(\frac{d t}{t}\right)}\|g\|_{L^{q}\left(\frac{d t}{t}\right)} .
$$

- For every $\phi \in L^{p}\left(d v_{n}\right) ; p \in[1,+\infty]$, the function $\phi_{t}$ belongs to $L^{p}\left(d v_{n}\right)$ and we have

$$
\left\|\phi_{t}\right\|_{p, v_{n}}=t^{-\frac{2 n+1}{p^{\prime}}}\|\phi\|_{p, v_{n}}
$$

where $p^{\prime}=p /(p-1)$.

- For all $\phi \in \mathrm{L}^{1}\left(\mathrm{~d} v_{\mathrm{n}}\right)$ and for every $(\mu, \lambda) \in \Gamma$,

$$
\mathscr{F}\left(\phi_{\mathrm{t}}\right)(\mu, \lambda)=\mathscr{F}(\phi)(\mathrm{t} \mu, \mathrm{t} \lambda)
$$

Definition 10. Let $p, q \in[1,+\infty], \gamma \in \mathbb{R}$ and $\phi \in S_{*, 0}^{1}\left(\mathbb{R} \times \mathbb{R}^{n}\right)$. We define the Besov space $\mathscr{B}_{\mathrm{p}, \mathrm{q}}^{\gamma, \phi}\left(\left[0,+\infty\left[\times \mathbb{R}^{\mathrm{n}}\right)\right.\right.$ to be the space of tempered distributions $f$ on $\mathbb{R} \times \mathbb{R}^{n}$, even with respect to the first variable and satisfying

- For all $t>0$, the function $f * \phi_{t}$ belongs to the space $L^{p}\left(d v_{n}\right)$.

- The function

$$
\mathrm{t} \longmapsto \frac{\left\|\mathrm{f} * \phi_{\mathrm{t}}\right\|_{p, v_{n}}}{\mathrm{t}^{\gamma}}
$$

belongs to the space $\mathrm{L}^{\mathrm{q}}\left(\frac{\mathrm{dt}}{\mathrm{t}}\right)$.

- The integral

$$
(\mathrm{r}, \mathrm{x}) \longmapsto \int_{0}^{+\infty} \mathrm{f} * \phi_{\mathrm{t}} * \phi_{\mathrm{t}}(\mathrm{r}, \mathrm{x}) \frac{\mathrm{dt}}{\mathrm{t}}
$$


is convergent in $S_{*}^{\prime}\left(\mathbb{R} \times \mathbb{R}^{n}\right)$ and

$$
f=\int_{0}^{+\infty} f * \phi_{t} * \phi_{t} \frac{d t}{t} .
$$

The space $\mathscr{B}_{p, q}^{\gamma, \Phi}\left(\left[0,+\infty\left[\times \mathbb{R}^{n}\right)\right.\right.$ is equipped with the norm

$$
M_{\mathfrak{p}, q}^{\gamma, \phi}(f)= \begin{cases}\left(\int_{0}^{+\infty}\left(\frac{\left\|f * \phi_{t}\right\|_{p}, v_{n}}{t^{\gamma}}\right)^{q} \frac{d t}{t}\right)^{\frac{1}{q}}, & \text { if } 1 \leqslant q<+\infty ; \\ \underset{t}{\operatorname{esssup}} \frac{\left\|f * \phi_{t}\right\|_{p, v_{n}}}{t^{\gamma}}, & \text { if } q=+\infty .\end{cases}
$$

Lemma 11. let $\psi \in S_{*}\left(\mathbb{R} \times \mathbb{R}^{n}\right)$ and let $\phi \in S_{*, 0}\left(\mathbb{R} \times \mathbb{R}^{n}\right)$. Then for all $k \in \mathbb{N}$, there exists $\phi_{\mathrm{k}} \in \mathrm{S}_{*, 0}\left(\mathbb{R} \times \mathbb{R}^{\mathrm{n}}\right)$ such that

$$
\psi * \phi_{\mathrm{t}}=\mathrm{t}^{2 \mathrm{k}}\left(\Delta^{\mathrm{k}} \psi\right) *\left(\phi_{\mathrm{k}}\right)_{\mathrm{t}},
$$

where $\Delta$ is the differential operator defined by

$$
\Delta=-\left(\frac{\partial^{2}}{\partial r^{2}}+\frac{n}{r} \frac{\partial}{\partial r}+\sum_{j=1}^{n}\left(\frac{\partial}{\partial x_{j}}\right)^{2}\right) .
$$

Moreover, for all $p \in[1,+\infty]$

$$
\left\|\psi * \phi_{t}\right\|_{p, v_{n}} \leqslant t^{2 k}\left\|\Delta^{k} \psi\right\|_{p, v_{n}}\left\|\phi_{k}\right\|_{1, v_{n}}
$$

and

$$
\left\|\psi * \phi_{t}\right\|_{p, v_{n}} \leqslant t^{-\frac{2 n+1}{p^{\prime}}}\|\psi\|_{1, v_{n}}\|\phi\|_{p, v_{n}} .
$$

Proof. The operator $\Delta$ is continuous from $S_{*}\left(\mathbb{R} \times \mathbb{R}^{n}\right)$ into itself and for all $\mathrm{f} \in \mathrm{S}_{*}\left(\mathbb{R} \times \mathbb{R}^{\mathfrak{n}}\right)$, we have

$$
\mathscr{F}(\Delta f)(\mu, \lambda)=\left(\mu^{2}+2|\lambda|^{2}\right) \mathscr{F}(f)(\mu, \lambda) .
$$

Let $\psi \in S_{*}\left(\mathbb{R} \times \mathbb{R}^{n}\right)$ and let $\phi \in S_{*, 0}\left(\mathbb{R} \times \mathbb{R}^{n}\right)$. From the relations (2.9) and (3.5), we get

$$
\begin{aligned}
\mathscr{F}\left(\psi * \phi_{\mathrm{t}}\right)(\mu, \lambda) & =\mathscr{F}(\psi)(\mu, \lambda) \mathscr{F}(\phi)(\mathrm{t} \mu, \mathrm{t} \lambda) \\
& =\mathrm{t}^{2}\left(\mu^{2}+2|\lambda|^{2}\right) \mathscr{F}(\psi)(\mu, \lambda) \frac{\mathscr{F}(\phi)(\mathrm{t} \mu, \mathrm{t} \lambda)}{\mathrm{t}^{2}\left(\mu^{2}+2|\lambda|^{2}\right)},
\end{aligned}
$$

and from the equality (3.9), we obtain

$$
\mathscr{F}\left(\psi * \phi_{\mathrm{t}}\right)(\mu, \lambda)=\mathrm{t}^{2} \mathscr{F}(\Delta \psi)(\mu, \lambda) \frac{\mathscr{F}(\phi)(\mathrm{t} \mu, \mathrm{t} \lambda)}{\mathrm{t}^{2}\left(\mu^{2}+2|\lambda|^{2}\right)} .
$$


Since, the function $\phi$ belongs to the space $S_{*, 0}\left(\mathbb{R} \times \mathbb{R}^{n}\right)$ then the function

$$
(\mu, \lambda) \longmapsto \frac{\mathscr{F}(\phi)(\mu, \lambda)}{\mu^{2}+2|\lambda|^{2}}
$$

belongs to the space $S_{*}(\Gamma)$ and from Theorem 5 , there exists $\phi_{1} \in S_{*}\left(\mathbb{R} \times \mathbb{R}^{n}\right)$ such that

$$
\mathscr{F}\left(\phi_{1}\right)(\mu, \lambda)=\frac{\mathscr{F}(\phi)(\mu, \lambda)}{\mu^{2}+2|\lambda|^{2}} .
$$

In particular, $\phi_{1}$ lies in $S_{*, 0}\left(\mathbb{R} \times \mathbb{R}^{n}\right)$ and the relation (3.10) leads to

$$
\mathscr{F}\left(\psi * \phi_{\mathrm{t}}\right)(\mu, \lambda)=\mathrm{t}^{2} \mathscr{F}(\Delta \psi)(\mu, \lambda) \mathscr{F}\left(\left(\phi_{1}\right)_{\mathrm{t}}\right)(\mu, \lambda),
$$

which implies that

$$
\psi * \phi_{\mathrm{t}}=\mathrm{t}^{2}(\Delta \psi) *\left(\phi_{1}\right)_{\mathrm{t}}
$$

By induction, for all $k \in \mathbb{N}^{*}$, there exists $\phi_{k} \in S_{*, 0}\left(\mathbb{R} \times \mathbb{R}^{n}\right)$ verifying

$$
\psi * \phi_{\mathrm{t}}=\mathrm{t}^{2 \mathrm{k}}\left(\Delta^{\mathrm{k}} \psi\right) *\left(\phi_{\mathrm{k}}\right)_{\mathrm{t}} .
$$

On the other hand, for every $t>0$ and by the relation (3.4), we get

$$
\begin{aligned}
\left\|\psi * \phi_{\mathrm{t}}\right\|_{p, v_{n}} & \leqslant\|\psi\|_{1, v_{n}}\left\|\phi_{\mathrm{t}}\right\|_{p, v_{n}} \\
& =\mathrm{t}^{-\frac{2 n+1}{p^{\prime}}}\|\psi\|_{1, v_{n}}\|\phi\|_{p, v_{n}}
\end{aligned}
$$

as the same way and using the relation (3.11), it follows that

$$
\left\|\psi * \phi_{t}\right\|_{p, v_{n}} \leqslant t^{2 k}\left\|\Delta^{k} \psi\right\|_{p, v_{n}}\left\|\phi_{k}\right\|_{1, v_{n}} .
$$

Proposition 12. Let $\phi \in S_{*, 0}^{1}\left(\mathbb{R} \times \mathbb{R}^{n}\right)$.

i) For all $f \in L^{2}\left(d v_{n}\right)$ we have

$$
f=\int_{0}^{+\infty} f * \phi_{t} * \phi_{t} \frac{d t}{t} ; \quad \text { in } L^{2}\left(d v_{n}\right) .
$$

ii) Let $\gamma \in \mathbb{R} ; \gamma<(2 n+1) / p$ and $f \in S_{*}^{\prime}\left(\mathbb{R} \times \mathbb{R}^{n}\right)$ such that for all $t>0$, the function $f * \phi_{\mathrm{t}}$ belongs to $\mathrm{L}^{\mathrm{p}}\left(\mathrm{d} v_{\mathrm{n}}\right)$ and the function

$$
\mathrm{t} \longmapsto \frac{\left\|\mathrm{f} * \phi_{\mathrm{t}}\right\|_{\mathrm{p}, v_{\mathrm{n}}}}{\mathrm{t}^{\gamma}}
$$

belongs to the space $L^{\mathrm{q}}\left(\frac{\mathrm{dt}}{\mathrm{t}}\right)$. Then the integral

$$
\int_{0}^{+\infty} f * \phi_{t} * \phi_{t} \frac{d t}{t}
$$

converges in $S_{*}^{\prime}\left(\mathbb{R} \times \mathbb{R}^{n}\right)$. 
Proof. i) Let $f \in \mathrm{L}^{2}\left(\mathrm{~d} v_{n}\right)$ and let $\mathrm{F}_{\mathrm{a}, \mathrm{b}}(\mathrm{f})$ be the function defined by

$$
\forall(r, x) \in\left[0,+\infty\left[\times \mathbb{R}^{n}, \quad F_{a, b}(f)(r, x)=\int_{a}^{b} f * \phi_{t} * \phi_{t}(r, x) \frac{d t}{t} ; \quad 0<a<b .\right.\right.
$$

The function $F_{a, b}(f)$ is well defined and by the relation (3.4) we have

$$
\begin{aligned}
\left|F_{a, b}(f)(r, x)\right| & \leqslant \int_{a}^{b}\|f\|_{2, v_{n}}\left\|\phi_{t} * \phi_{t}\right\|_{2, v_{n}} \frac{d t}{t} \\
& \leqslant\|f\|_{2, v_{n}} \int_{a}^{b}\left\|\phi_{t}\right\|_{1, v_{n}}\left\|\phi_{t}\right\|_{2, v_{n}} \frac{d t}{t} \\
& \leqslant\|f\|_{2, v_{n}}\|\phi\|_{1, v_{n}}\|\phi\|_{2, v_{n}} \int_{a}^{b} t^{-\frac{2 n+1}{2}-1} d t \\
& <+\infty .
\end{aligned}
$$

Moreover, the function $F_{a, b}(f)$ belongs to $L^{2}\left(d v_{n}\right)$. Indeed by Minkowski's inequality [12] and the relation (3.4) we get

$$
\begin{aligned}
\left\|F_{a, b}(f)\right\|_{2, v_{n}} & \leqslant \int_{a}^{b}\left\|f * \phi_{t} * \phi_{t}\right\|_{2, v_{n}} \frac{d t}{t} \\
& \leqslant \int_{a}^{b}\|f\|_{2, v_{n}}\left\|\phi_{t}\right\|_{1, v_{n}}^{2} \frac{d t}{t} \\
& =\|f\|_{2, v_{n}}\|\phi\|_{1, v_{n}}^{2} \log \left(\frac{b}{a}\right) \\
& <+\infty .
\end{aligned}
$$

On the other hand, by Fubini's theorem and the relation (3.5), we have

$$
\mathscr{F}\left(\mathrm{F}_{\mathrm{a}, \mathrm{b}}(\mathrm{f})\right)(\mu, \lambda)=\mathscr{F}(\mathrm{f})(\mu, \lambda) \int_{\mathrm{a}}^{\mathrm{b}}(\mathscr{F}(\phi)(\mathrm{t} \mu, \mathrm{t} \lambda))^{2} \frac{\mathrm{dt}}{\mathrm{t}} .
$$

Thus, by the Plancherel theorem

$$
\begin{aligned}
\left\|f-F_{a, b}(f)\right\|_{2, v_{n}}^{2} & =\left\|\mathscr{F}(f)-\mathscr{F}\left(F_{a, b}(f)\right)\right\|_{2, \gamma_{n}}^{2} \\
& =\iint_{\Gamma_{+}}|\mathscr{F}(f)(\mu, \lambda)|^{2}\left|1-\int_{a}^{b}(\mathscr{F}(\phi)(t \mu, t \lambda))^{2} \frac{d t}{t}\right| d \gamma_{n}(\mu, \lambda) .
\end{aligned}
$$

Using the fact that

$$
\int_{0}^{+\infty}(\mathscr{F}(\phi)(\mathrm{t} \mu, \mathrm{t} \lambda))^{2} \frac{\mathrm{dt}}{\mathrm{t}}=1
$$

we have

$$
\forall(\mu, \lambda) \in \Gamma \backslash\{(0,0)\}, \quad\left|1-\int_{\mathrm{a}}^{\mathrm{b}}(\mathscr{F}(\phi)(\mathrm{t} \mu, \mathrm{t} \lambda))^{2} \frac{\mathrm{dt}}{\mathrm{t}}\right| \leqslant 1
$$

and applying the dominated convergence theorem, we deduce that

$$
\lim _{\substack{a \rightarrow 0^{+} \\ b \rightarrow+\infty}}\left\|f-F_{a, b}(f)\right\|_{2, v_{n}}=0
$$


ii) Let $f$ be in $S_{*}^{\prime}\left(\mathbb{R} \times \mathbb{R}^{n}\right)$ satisfying the hypothesis, then the function $F_{a, b}(f)$ defined above is bounded on $\mathbb{R} \times \mathbb{R}^{n}$. In fact

$$
\begin{aligned}
\left|F_{a, b}(f)(r, x)\right| & \leqslant \int_{a}^{b}\left\|f * \phi_{t}\right\|_{p, v_{n}}\left\|\phi_{t}\right\|_{p^{\prime}, v_{n}} \frac{d t}{t} \\
& =\|\phi\|_{p^{\prime}, v_{n}} \int_{a}^{b} \frac{\left\|f * \phi_{t}\right\|_{p, v_{n}}}{t^{\gamma}} t^{-\frac{2 n+1}{p}+\gamma} \frac{d t}{t} \\
& \leqslant\|\phi\|_{p^{\prime}, v_{n}}\left[\int_{a}^{b}\left(\frac{\left\|f * \phi_{t}\right\|_{p, v_{n}}}{t^{\gamma}}\right)^{q} \frac{d t}{t}\right]^{\frac{1}{q}}\left[\int_{a}^{b} t^{\left(-\frac{2 n+1}{p}+\gamma\right)} q^{\prime} \frac{d t}{t}\right]^{\frac{1}{q^{\prime}}} \\
& <+\infty,
\end{aligned}
$$

where $\mathrm{q}^{\prime}$ is the conjugate exponent of $\mathrm{q}$.

Thus for all $a, b \in \mathbb{R} ; b>a>0$, the function $F_{a, b}(f)$ defines an element of $S_{*}^{\prime}\left(\mathbb{R} \times \mathbb{R}^{n}\right)$.

Let $\psi \in S_{*}\left(\mathbb{R} \times \mathbb{R}^{n}\right)$, by Fubini's theorem, we have

$$
\begin{aligned}
& \left\langle F_{a, b}(f), \psi\right\rangle=\int_{0}^{+\infty} \int_{\mathbb{R}^{n}}\left\{\int_{a}^{b} f * \phi_{t} * \phi_{t}(r, x) \psi(r, x) \frac{d t}{t}\right\} d v_{n}(r, x) \\
& =\int_{a}^{b}\left\{\int_{0}^{+\infty} \int_{\mathbb{R}^{n}} f * \phi_{t} * \phi_{t}(r, x) \psi(r, x) d v_{n}(r, x)\right\} \frac{d t}{t} \\
& =\int_{a}^{b}\left\{\int_{0}^{+\infty} \int_{\mathbb{R}^{n}} \psi(r, x)\left[\int_{0}^{+\infty} \int_{\mathbb{R}^{n}} f * \phi_{t}(s, y) \tau_{(r,-x)}\left(\check{\phi}_{t}\right)(s, y) d v_{n}(s, y)\right] d v_{n}(r, x)\right\} \frac{d t}{t} \\
& =\int_{a}^{b}\left\{\int_{0}^{+\infty} \int_{\mathbb{R}^{n}} f * \phi_{t}(s, y)\left[\int_{0}^{+\infty} \int_{\mathbb{R}^{n}} \psi(r, x) \tau_{(s,-y)}\left(\phi_{t}\right)(r, x) d v_{n}(r, x)\right] d v_{n}(s, y)\right\} \frac{d t}{t} \\
& =\int_{a}^{b}\left[\int_{0}^{+\infty} \int_{\mathbb{R}^{n}} f * \phi_{t}(s, y) \check{\phi}_{t} * \psi(s, y) d v_{n}(s, y)\right] \frac{d t}{t} .
\end{aligned}
$$

However,

$$
\begin{aligned}
\int_{0}^{+\infty}\left[\int_{0}^{+\infty} \int_{\mathbb{R}^{n}}\left|f * \phi_{\mathrm{t}}(s, y)\right|\left|\check{\phi}_{\mathrm{t}} * \psi(s, y)\right| \mathrm{d} v_{\mathrm{n}}(\mathrm{s}, \mathrm{y})\right] \frac{\mathrm{dt}}{\mathrm{t}} \\
\quad \leqslant \int_{0}^{+\infty}\left\|\mathrm{f} * \phi_{\mathrm{t}}\right\|_{p, v_{n}}\left\|\check{\phi}_{\mathrm{t}} * \psi\right\|_{p^{\prime}, v_{n}} \frac{\mathrm{dt}}{\mathrm{t}} \\
\quad \leqslant \int_{0}^{1}\left\|\mathrm{f} * \phi_{\mathrm{t}}\right\|_{p, v_{n}}\left\|\check{\phi}_{\mathrm{t}} * \psi\right\|_{p^{\prime}, v_{n}} \frac{\mathrm{dt}}{\mathrm{t}}+\int_{1}^{+\infty}\left\|f * \phi_{\mathrm{t}}\right\|_{p, v_{n}}\left\|\check{\phi}_{\mathrm{t}} * \psi\right\|_{p^{\prime}, v_{n}} \frac{\mathrm{dt}}{\mathrm{t}} .
\end{aligned}
$$


Using the relations (3.7) and (3.8), we get

$$
\begin{aligned}
& \int_{0}^{+\infty}\left\{\int_{0}^{+\infty} \int_{\mathbb{R}^{n}}\left|f * \phi_{t}(s, y)\right|\left|\check{\phi}_{t} * \psi(s, y)\right| d v_{n}(s, y)\right\} \frac{d t}{t} \\
& \leqslant\left\|\Delta^{k} \psi\right\|_{p^{\prime}, v_{n}}\left\|\phi_{k}\right\|_{1, v_{n}} \int_{0}^{1} t^{2 k}\left\|f * \phi_{t}\right\|_{p, v_{n}} \frac{d t}{t} \\
& +\|\psi\|_{1, v_{n}}\|\phi\|_{p^{\prime}, v_{n}} \int_{1}^{+\infty} t^{-\frac{2 n+1}{p}}\left\|f * \phi_{t}\right\|_{p, v_{n}} \frac{d t}{t} \\
& \left\|\Delta^{k} \psi\right\|_{p^{\prime}, v_{n}}\left\|\phi_{k}\right\|_{1, v_{n}} \int_{0}^{+\infty} t^{2 k+\gamma} \mathbf{1}_{[0,1]}(t) \frac{\left\|f * \phi_{t}\right\|_{p, v_{n}}}{t^{\gamma}} \frac{d t}{t} \\
& +\|\psi\|_{1, v_{n}}\|\phi\|_{p^{\prime}, v_{n}} \int_{0}^{+\infty} t^{-\frac{2 n+1}{p}+\gamma} \mathbf{1}_{[1,+\infty[}(t) \frac{\left\|f * \phi_{t}\right\|_{p, v_{n}}}{t^{\gamma}} \frac{d t}{t} .
\end{aligned}
$$

Let $k$ be sufficiently large. Using the hypothesis $\gamma<(2 n+1) / p$ and applying Hölder's inequality, we obtain

$$
\begin{aligned}
& \int_{0}^{+\infty}\left\{\int_{0}^{+\infty} \int_{\mathbb{R}^{n}}\left|f * \phi_{t}(s, y)\right|\left|\check{\phi}_{t} * \psi(s, y)\right| d v_{n}(s, y)\right\} \frac{d t}{t} \\
& \leqslant\left\|\Delta^{k} \psi\right\|_{p^{\prime}, v_{n}}\left\|\phi_{k}\right\|_{1, v_{n}}\left\|t^{2 k+\gamma} \mathbf{1}_{[0,1]}\right\|_{L^{q^{\prime}\left(\frac{d t}{t}\right)}}\left\|\frac{\left\|f * \phi_{t}\right\|_{p, v_{n}}}{t^{\gamma}}\right\|_{L^{q}\left(\frac{d t}{t}\right)} \\
& +\|\psi\|_{1, v_{n}}\|\phi\|_{p^{\prime}, v_{n}}\left\|t^{-\frac{2 n+1}{p}+\gamma} \mathbf{1}_{[1,+\infty[}\right\|_{L^{q^{\prime}\left(\frac{\mathrm{d} t}{\mathrm{t}}\right)}}\left\|\frac{\left\|f * \phi_{\mathrm{t}}\right\|_{p, v_{n}}}{\mathrm{t}^{\gamma}}\right\|_{\mathrm{L}^{\mathrm{q}\left(\frac{\mathrm{d} t}{\mathrm{t}}\right)}} \\
& <+\infty \text {. }
\end{aligned}
$$

This shows that for all $\psi \in S_{*}\left(\mathbb{R} \times \mathbb{R}^{n}\right), \underset{\substack{a \rightarrow 0^{+} \\ b \rightarrow+\infty}}{\lim _{a, b}}\left\langle F_{a}(f), \psi\right\rangle$ exists and

$$
\lim _{\substack{a \rightarrow 0^{+} \\ b \rightarrow+\infty}}\left\langle F_{a, b}(f), \psi\right\rangle=\int_{0}^{+\infty} \int_{0}^{+\infty} \int_{\mathbb{R}^{n}} f * \phi_{t}(s, y) \check{\phi}_{t} * \psi(s, y) d v_{n}(s, y) \frac{d t}{t} .
$$

This means that the integral

$$
\int_{0}^{+\infty} \mathrm{f} * \phi_{\mathrm{t}} * \phi_{\mathrm{t}} \frac{\mathrm{dt}}{\mathrm{t}}
$$

converges in $S_{*}^{\prime}\left(\mathbb{R} \times \mathbb{R}^{n}\right)$.

Lemma 13. 1) Let $f \in \mathscr{B}_{\mathrm{p}, \mathrm{q}}^{\gamma, \phi}\left(\left[0,+\infty\left[\times \mathbb{R}^{n}\right)\right.\right.$. Then

i) For all $\psi \in S_{*}\left(\mathbb{R} \times \mathbb{R}^{n}\right)$, we have

$$
f * \psi=\int_{0}^{\infty} f * \phi_{t} * \phi_{t} * \psi \frac{d t}{t} .
$$

ii) For all $\psi \in S_{*, 0}^{1}\left(\mathbb{R} \times \mathbb{R}^{n}\right)$,

$$
f=\int_{0}^{+\infty} f * \psi_{\rho} * \psi_{\rho} \frac{d \rho}{\rho} .
$$


2) For all $g \in S_{*}\left(\mathbb{R} \times \mathbb{R}^{n}\right)$ and for all $\psi \in S_{*, 0}^{1}\left(\mathbb{R} \times \mathbb{R}^{n}\right)$, we have

$$
\int_{0}^{+\infty} g * \psi_{\rho} * \psi_{\rho} \frac{\mathrm{d} \rho}{\rho}=\mathrm{g}
$$

Proof. 1) Let $f \in \mathscr{B}_{\mathbf{p}, \boldsymbol{q}}^{\gamma, \Phi}\left(\left[0,+\infty\left[\times \mathbb{R}^{n}\right)\right.\right.$.

i) For every $\psi \in S_{*}\left(\mathbb{R} \times \mathbb{R}^{n}\right)$, we have

$$
\begin{aligned}
f * \psi(r, x) & =\left\langle f, \tau_{(r,-x)} \check{\psi}\right\rangle \\
& =\lim _{\substack{a \rightarrow 0^{+} \\
b \rightarrow+\infty}}\left\langle\int_{a}^{b} f * \phi_{t} * \phi_{t} \frac{d t}{t}, \tau_{(r,-x)} \check{\psi}\right\rangle \\
& =\lim _{\substack{a \rightarrow 0^{+} \\
b \rightarrow+\infty}} \int_{0}^{+\infty} \int_{\mathbb{R}^{n}}\left(\int_{a}^{b} f * \phi_{t} * \phi_{t}(s, y) \frac{d t}{t}\right) \tau_{(r,-x)} \check{\psi}(s, y) d v_{n}(s, y),
\end{aligned}
$$

and by Fubini's theorem, we obtain

$$
\begin{aligned}
f * \psi(r, x) & =\lim _{\substack{a \rightarrow 0^{+} \\
b \rightarrow+\infty}} \int_{a}^{b}\left(\int_{0}^{+\infty} \int_{\mathbb{R}^{n}} f * \phi_{t} * \phi_{t}(s, y) \tau_{(r,-x)} \check{\psi}(s, y) d v_{n}(s, y)\right) \frac{d t}{t} \\
& =\lim _{\substack{a \rightarrow 0^{+} \\
b \rightarrow+\infty}} \int_{a}^{b} f * \phi_{t} * \phi_{t} * \psi(r, x) \frac{d t}{t} \\
& =\int_{0}^{+\infty} f * \phi_{t} * \phi_{t} * \psi(r, x) \frac{d t}{t}
\end{aligned}
$$

ii) Let $\psi \in S_{*, 0}^{1}\left(\mathbb{R} \times \mathbb{R}^{n}\right)$. For all positive real number $\rho$, we have

$$
\psi_{\rho} * \psi_{\rho}=(\psi * \psi)_{\rho} .
$$

Applying i) we get

$$
\mathrm{f} * \psi_{\rho} * \psi_{\rho}=\int_{0}^{+\infty} \mathrm{f} * \phi_{\mathrm{t}} * \phi_{\mathrm{t}} * \psi_{\rho} * \psi_{\rho} \frac{\mathrm{dt}}{\mathrm{t}} .
$$

Now, let $a_{1}, a_{2}, b_{1}, b_{2}$ be positive real numbers such that

$$
\mathscr{F}(\phi)(\mu, \lambda)=0 \quad \text { if } \mu^{2}+2|\lambda|^{2}<a_{1}^{2} \quad \text { or } \mu^{2}+2|\lambda|^{2}>b_{1}^{2}
$$

and

$$
\mathscr{F}(\psi)(\mu, \lambda)=0 \quad \text { if } \quad \mu^{2}+2|\lambda|^{2}<a_{2}^{2} \quad \text { or } \quad \mu^{2}+2|\lambda|^{2}>b_{2}^{2}
$$

then

$$
\mathscr{F}\left(\phi_{\mathrm{t}}\right)(\mu, \lambda) \mathscr{F}\left(\psi_{\rho}\right)(\mu, \lambda)=0 \quad \text { if } \quad \frac{\mathrm{t}}{\rho} \notin\left[\frac{\mathrm{a}_{1}}{\mathrm{~b}_{2}}, \frac{\mathrm{b}_{1}}{\mathrm{a}_{2}}\right]=[\alpha, \beta],
$$

and consequently, by the relation (2.9) and Theorem 4

$$
\phi_{\mathrm{t}} * \psi_{\rho}=0 \quad \text { if } \quad \frac{\mathrm{t}}{\rho} \notin[\alpha, \beta] .
$$


Thus,

$$
\mathrm{f} * \psi_{\rho} * \psi_{\rho}=\int_{\rho \alpha}^{\rho \beta} \mathrm{f} * \phi_{\mathrm{t}} * \phi_{\mathrm{t}} * \psi_{\rho} * \psi_{\rho} \frac{\mathrm{dt}}{\mathrm{t}}
$$

So for all $a, b \in \mathbb{R} ; 0<a<b$,

$$
\int_{a}^{b} f * \psi_{\rho} * \psi_{\rho} \frac{d \rho}{\rho}=\int_{a}^{b}\left(\int_{\rho \alpha}^{\rho \beta} f * \phi_{t} * \phi_{t} * \psi_{\rho} * \psi_{\rho} \frac{d t}{t}\right) \frac{d \rho}{\rho} .
$$

By Fubini's theorem, we get

$$
\int_{a}^{b} f * \psi_{\rho} * \psi_{\rho} \frac{d \rho}{\rho}=\int_{a \alpha}^{b \beta}\left(\int_{\frac{t}{\beta}}^{\frac{t}{\alpha}} f * \phi_{t} * \phi_{t} * \psi_{\rho} * \psi_{\rho} \frac{d \rho}{\rho}\right) \frac{d t}{t} .
$$

On the other hand, we have

$$
\begin{array}{rl}
\int_{\frac{\mathrm{t}}{\beta}}^{\frac{\mathrm{t}}{\alpha}} \mathrm{f} & * \phi_{\mathrm{t}} * \phi_{\mathrm{t}} * \psi_{\rho} * \psi_{\rho}(\mathrm{r}, \mathrm{x}) \frac{\mathrm{d} \rho}{\rho} \\
& =\int_{\frac{\mathrm{t}}{\beta}}^{\frac{\mathrm{t}}{\alpha}}\left(\int_{0}^{+\infty} \int_{\mathbb{R}^{n}} \phi_{\mathrm{t}} * \psi_{\rho} * \psi_{\rho}(\mathrm{s}, \mathrm{y}) \tau_{(\mathrm{r},-x)}\left(\mathrm{f} * \phi_{\mathrm{t}}\right)(\mathrm{s}, \mathrm{y}) \mathrm{d} v_{\mathrm{n}}(\mathrm{s}, \mathrm{y})\right) \frac{\mathrm{d} \rho}{\rho} \\
& =\int_{0}^{+\infty} \int_{\mathbb{R}^{n}} \tau_{(\mathrm{r},-x)}\left(\mathrm{f} * \phi_{\mathrm{t}}\right)(\mathrm{s}, \mathrm{y})\left(\int_{\frac{\mathrm{t}}{\beta}}^{\frac{\mathrm{t}}{\alpha}} \phi_{\mathrm{t}} * \psi_{\rho} * \psi_{\rho}(\mathrm{s}, \mathrm{y}) \frac{\mathrm{d} \rho}{\rho}\right) \mathrm{d} v_{\mathrm{n}}(\mathrm{s}, \mathrm{y}) \\
& =\int_{0}^{+\infty} \int_{\mathbb{R}^{n}} \tau_{(\mathrm{r},-x)}\left(\mathrm{f} * \phi_{\mathrm{t}}\right)(\mathrm{s}, \mathrm{y})\left(\int_{0}^{+\infty} \phi_{\mathrm{t}} * \psi_{\rho} * \psi_{\rho}(\mathrm{s}, \mathrm{y}) \frac{\mathrm{d} \rho}{\rho}\right) \mathrm{d} v_{\mathrm{n}}(\mathrm{s}, \mathrm{y}) .
\end{array}
$$

However by i) of Proposition 12 it follows that

$$
\begin{aligned}
\int_{\frac{\mathrm{t}}{\beta}}^{\frac{\mathrm{t}}{\alpha}} \mathrm{f} * \phi_{\mathrm{t}} * \phi_{\mathrm{t}} * \psi_{\rho} * \psi_{\rho}(\mathrm{r}, \mathrm{x}) \frac{\mathrm{d} \rho}{\rho} & =\int_{0}^{+\infty} \int_{\mathbb{R}^{n}} \phi_{\mathrm{t}}(\mathrm{s}, \mathrm{y}) \tau_{(\mathrm{r},-x)}\left(\mathrm{f} * \phi_{\mathrm{t}}\right)(\mathrm{s}, \mathrm{y}) \mathrm{d} v_{\mathrm{n}}(\mathrm{s}, \mathrm{y}) \\
& =\mathrm{f} * \phi_{\mathrm{t}} * \phi_{\mathrm{t}}(\mathrm{r}, \mathrm{x}) .
\end{aligned}
$$

Replacing in the equality (3.14), we obtain

$$
\int_{a}^{b} f * \psi_{\rho} * \psi_{\rho} \frac{d \rho}{\rho}=\int_{a \alpha}^{b \beta} f * \phi_{t} * \phi_{t} \frac{d t}{t} .
$$

2) We know that for all $g \in S_{*}\left(\mathbb{R} \times \mathbb{R}^{n}\right)$ and $\psi \in S_{*, 0}^{1}\left(\mathbb{R} \times \mathbb{R}^{n}\right)$, the function $\mathrm{g} * \psi_{\rho} * \psi_{\rho}$ belongs to the space $S_{*}\left(\mathbb{R} \times \mathbb{R}^{n}\right)$. By Theorem 4 and the relation (3.5), we have

$$
\mathrm{g} * \psi_{\rho} * \psi_{\rho}(\mathrm{r}, \mathrm{x})=\iint_{\Gamma_{+}} \mathscr{F}(\mathrm{g})(\mu, \lambda)(\mathscr{F}(\psi)(\rho \mu, \rho \lambda))^{2} \overline{\varphi_{\mu, \lambda}(\mathrm{r}, x)} \mathrm{d} \gamma_{\mathrm{n}}(\mu, \lambda)
$$

then

$$
\int_{0}^{+\infty} \mathrm{g} * \psi_{\rho} * \psi_{\rho}(\mathrm{r}, \mathrm{x}) \frac{\mathrm{d} \rho}{\rho} \iint_{\Gamma_{+}} \mathscr{F}(\mathrm{g})(\mu, \lambda) \overline{\varphi_{\mu, \lambda}(\mathrm{r}, x)}\left[\int_{0}^{+\infty}(\mathscr{F}(\psi)(\rho \mu, \rho \lambda))^{2} \frac{\mathrm{d} \rho}{\rho}\right] \mathrm{d} \gamma_{\mathrm{n}}(\mu, \lambda)
$$


and by the relation (3.1) and Theorem 4, we get

$$
\begin{aligned}
\int_{0}^{+\infty} \mathrm{g} * \psi_{\rho} * \psi_{\rho}(\mathrm{r}, x) \frac{\mathrm{d} \rho}{\rho} & =\iint_{\Gamma_{+}} \mathscr{F}(\mathrm{g})(\mu, \lambda) \overline{\varphi_{\mu, \lambda}(\mathrm{r}, x)} \mathrm{d} \gamma_{\mathrm{n}}(\mu, \lambda) \\
& =\mathrm{g}(\mathrm{r}, \mathrm{x}) .
\end{aligned}
$$

Theorem 14. Let $p, q \in[1,+\infty]$ and $\gamma \in \mathbb{R}$, the space $\mathscr{B}_{p, q}^{\gamma, \phi}\left(\left[0,+\infty\left[\times \mathbb{R}^{n}\right)\right.\right.$ is independent of the choice of the function $\phi$ in $S_{*, 0}^{1}\left(\mathbb{R} \times \mathbb{R}^{n}\right)$ and will be denoted by $\mathscr{B}_{\mathrm{p}, \mathrm{q}}^{\gamma}\left(\left[0,+\infty\left[\times \mathbb{R}^{n}\right)\right.\right.$.

Proof. Let $\mathrm{f} \in \mathscr{B}_{\mathrm{p}, \mathrm{q}}^{\gamma, \phi}\left(\left[0,+\infty\left[\times \mathbb{R}^{\mathrm{n}}\right)\right.\right.$ and let $\psi \in \mathrm{S}_{*, 0}^{1}\left(\mathbb{R} \times \mathbb{R}^{\mathrm{n}}\right)$. From Lemma 13 and the relation (3.13), we have

$$
\begin{aligned}
f * \psi_{\rho} & =\int_{0}^{+\infty} f * \phi_{\mathrm{t}} * \phi_{\mathrm{t}} * \psi_{\rho} \frac{\mathrm{dt}}{\mathrm{t}} \\
& =\int_{\rho \alpha}^{\rho \beta} \mathrm{f} * \phi_{\mathrm{t}} * \phi_{\mathrm{t}} * \psi_{\rho} \frac{\mathrm{dt}}{\mathrm{t}} \\
& =\int_{\alpha}^{\beta} \mathrm{f} * \phi_{\rho \mathrm{s}} * \phi_{\rho \mathrm{s}} * \psi_{\rho} \frac{\mathrm{ds}}{\mathrm{s}} .
\end{aligned}
$$

Thus, from Minkowski's inequality and the relations (2.7) and (3.4), we get

$$
\begin{aligned}
\left\|f * \psi_{\rho}\right\|_{p, v_{n}} & \leqslant \int_{\alpha}^{\beta}\left\|f * \phi_{\rho s} * \psi_{\rho} * \phi_{\rho s}\right\|_{p, v_{n}} \frac{\mathrm{ds}}{\mathrm{s}} \\
& \leqslant \int_{\alpha}^{\beta}\left\|f * \phi_{\rho s}\right\|_{p, v_{n}}\left\|\psi_{\rho} * \phi_{\rho s}\right\|_{1, v_{n}} \frac{\mathrm{d} s}{\mathrm{~s}} \\
& \leqslant\|\psi\|_{1, v_{n}}\|\phi\|_{1, v_{n}} \int_{\alpha}^{\beta}\left\|f * \phi_{\rho s}\right\|_{p, v_{n}} \frac{\mathrm{d} s}{\mathrm{~s}},
\end{aligned}
$$

and by Hölder's inequality, it follows that

$$
\begin{aligned}
\left\|f * \psi_{\rho}\right\|_{p, v_{n}} & \leqslant\|\psi\|_{1, v_{n}}\|\phi\|_{1, v_{n}}\left(\int_{\alpha}^{\beta}\left(\frac{\left\|f * \phi_{\rho s}\right\|_{p, v_{n}}}{(\rho s)^{\gamma}}\right)^{q} \frac{d s}{s}\right)^{\frac{1}{q}}\left(\int_{\alpha}^{\beta}(\rho s)^{\gamma q^{\prime}} \frac{d s}{s}\right)^{\frac{1}{q^{\prime}}} \\
& \leqslant\|\psi\|_{1, v_{n}}\|\phi\|_{1, v_{n}} M_{p, q}^{\gamma, \phi}(f) \rho^{\gamma}\left(\int_{\alpha}^{\beta} s^{\gamma} q^{\prime} \frac{d s}{s}\right)^{\frac{1}{q^{\prime}}} \\
& <+\infty
\end{aligned}
$$

where $\mathrm{q}^{\prime}$ is the conjugate exponent of q. Now, by the relation (3.15), we have

$$
\begin{aligned}
\frac{\left\|f * \psi_{\rho}\right\|_{p, v_{n}}}{\rho^{\gamma}} & \leqslant\|\psi\|_{1, v_{n}}\|\phi\|_{1, v_{n}} \int_{\alpha}^{\beta} \frac{s^{\gamma} \frac{\left\|f * \phi_{\rho s}\right\|_{p, v_{n}}}{(\rho s)^{\gamma}} \frac{d s}{s}}{} \leqslant\|\psi\|_{1, v_{n}}\|\phi\|_{1, v_{n}} \int_{\frac{1}{\beta}}^{\frac{1}{\alpha}} t^{-\gamma} \frac{\left\|f * \phi_{\frac{\rho}{t}}\right\|_{p, v_{n}}}{\left(\frac{\rho}{t}\right)^{\gamma}} \frac{d t}{t} \\
& =\|\psi\|_{1, v_{n}}\|\phi\|_{1, v_{n}}\left[t^{-\gamma} 1_{\left[\frac{1}{\beta}, \frac{1}{\alpha}\right]} \star\left(\frac{\left\|f * \phi_{t}\right\|_{p, v_{n}}}{t^{\gamma}}\right)\right](\rho),
\end{aligned}
$$


where $\star$ is the convolution product defined on $] 0,+\infty[$ by the relation (3.2). By the relation (3.3), we obtain

$$
\begin{aligned}
M_{p, q}^{\gamma, \psi}(f) & \leqslant\|\psi\|_{1, v_{n}}\|\phi\|_{1, v_{n}}\left\|t^{-\gamma} \mathbf{1}_{\left[\frac{1}{\beta}, \frac{1}{\alpha}\right]}\right\|_{L^{1}\left(\frac{d t}{t}\right)} M_{p, q}^{\gamma, \phi}(f) \\
& <+\infty
\end{aligned}
$$

and the proof is complete if we take into account Lemma 13.

Proposition 15. Let $\mathrm{p}, \mathrm{q} \in[1,+\infty]$ and $\gamma \in \mathbb{R}$. The Besov space

$\mathscr{B}_{\mathrm{p}, \mathrm{q}}^{\gamma}\left(\left[0,+\infty\left[\times \mathbb{R}^{n}\right)\right.\right.$ is homogeneous of degree equal to $(2 n+1) / p-\gamma-2 n-1$, that is for every $f \in \mathscr{B}_{p, q}^{\gamma}\left(\left[0,+\infty\left[\times \mathbb{R}^{n}\right)\right.\right.$ and $t>0$, the distribution $f_{t}$ belongs to the space $\mathscr{B}_{\mathrm{p}, \mathrm{q}}^{\gamma}\left(\left[0,+\infty\left[\times \mathbb{R}^{\mathrm{n}}\right)\right.\right.$ and we have

$$
M_{p, q}^{\gamma, \phi}\left(f_{t}\right)=t^{\frac{2 n+1}{p}-\gamma-2 n-1} M_{p, q}^{\gamma, \phi}(f),
$$

where

$$
\left\langle f_{t}, \varphi\right\rangle=\left\langle f, \frac{1}{t^{2 n+1}} \varphi_{\frac{1}{t}}\right\rangle ; \quad \varphi \in S_{*}\left(\mathbb{R} \times \mathbb{R}^{n}\right) .
$$

Proof. Let $\phi \in S_{*, 0}^{1}\left(\mathbb{R} \times \mathbb{R}^{n}\right)$, we have

$$
\begin{aligned}
f_{t} * \phi_{\rho}(r, x) & =\left\langle f_{t}, \tau_{(r,-x)}\left(\check{\phi}_{\rho}\right)\right\rangle \\
& =\left\langle f, \frac{1}{t^{2 n+1}}\left(\tau_{(r,-x)}\left(\check{\phi}_{\rho}\right)\right)_{\frac{1}{t}}\right\rangle .
\end{aligned}
$$

However,

$$
\begin{aligned}
\frac{1}{\mathrm{t}^{2 \mathrm{n}+1}}\left(\tau_{(\mathrm{r},-x)}\left(\check{\phi}_{\rho}\right)\right)_{\frac{1}{\mathrm{t}}}(s, y) & =\tau_{(\mathrm{r},-x)}\left(\check{\phi}_{\rho}\right)(\mathrm{t} s, \mathrm{ty}) \\
& =\frac{1}{\mathrm{t}^{2 \mathrm{n}+1}} \tau_{\left(\frac{\mathrm{r}}{\mathrm{t}},-\frac{\mathrm{x}}{\mathrm{t}}\right)}\left(\check{\phi}_{\frac{\rho}{\mathrm{t}}}\right)(\mathrm{s}, \mathrm{y})
\end{aligned}
$$

consequently,

$$
\begin{aligned}
f_{t} * \phi_{\rho}(r, x) & =\left\langle f, \frac{1}{t^{2 n+1}} \tau_{\left(\frac{r}{t},-\frac{x}{t}\right)}\left(\check{\phi}_{\frac{\rho}{t}}\right)\right\rangle \\
& =\left(f * \phi_{\frac{\rho}{t}}\right)_{t}(r, x) .
\end{aligned}
$$

Hence, from the relation (3.4), we get

$$
\left\|f_{t} * \phi_{\rho}\right\|_{p, v_{n}}=t^{-\frac{2 n+1}{p^{\prime}}}\left\|f * \phi_{\frac{\rho}{t}}\right\|_{p, v_{n}},
$$

this shows that for all $\rho>0$, the function $f_{t} * \phi_{\rho}$ belongs to $L^{p}\left(d v_{n}\right)$ and we have

$$
\begin{aligned}
\left\|\frac{\left\|f_{t} * \phi_{\rho}\right\|_{p, v_{n}}}{\rho^{\gamma}}\right\|_{L^{q}\left(\frac{d \rho}{\rho}\right)}^{q} & =t^{-\frac{2 n+1}{p^{\prime}}} q \int_{0}^{+\infty}\left(\frac{\left\|f * \phi_{\frac{\rho}{t}}\right\|_{p, v_{n}}}{\rho^{\gamma}}\right)^{q} \frac{d \rho}{\rho} \\
& =t^{-\frac{2 n+1}{p^{\prime}} q} t^{-\gamma q} \int_{0}^{+\infty}\left(\frac{\left\|f * \phi_{s}\right\|_{p, v_{n}}}{s^{\gamma}}\right)^{q} \frac{d s}{s} \\
& =t^{-q\left(\frac{2 n+1}{p^{\prime}}+\gamma\right)}\left[M_{p, q}^{\gamma, \phi}(f)\right]^{q},
\end{aligned}
$$


which proves that the function

$$
\rho \longmapsto \frac{\left\|f_{t} * \phi_{\rho}\right\|_{p, v_{n}}}{\rho^{\gamma}}
$$

belongs to the space $\mathrm{L}^{\mathrm{q}}\left(\frac{\mathrm{d} \rho}{\rho}\right)$ and that

$$
M_{p, q}^{\gamma, \phi}\left(f_{t}\right)=t^{\frac{2 n+1}{p}-\gamma-2 n-1} M_{p, q}^{\gamma, \phi}(f) .
$$

On the other hand, from the relations (3.12) and (3.16), we have

$$
\begin{aligned}
\int_{0}^{+\infty} \mathrm{f}_{\mathrm{t}} * \phi_{\rho} * \phi_{\rho}(\mathrm{r}, \mathrm{x}) \frac{\mathrm{d} \rho}{\rho} & \frac{1}{\mathrm{t}^{2 \mathrm{n}+1}} \int_{0}^{+\infty} \mathrm{f} *(\phi * \phi)_{\mathrm{s}}\left(\frac{\mathrm{r}}{\mathrm{t}}, \frac{x}{\mathrm{t}}\right) \frac{\mathrm{ds}}{\mathrm{s}} \\
= & \frac{1}{\mathrm{t}^{2 \mathrm{n}+1}} \int_{0}^{+\infty} \mathrm{f} * \phi_{\mathrm{s}} * \phi_{\mathrm{s}}\left(\frac{r}{\mathrm{t}}, \frac{x}{\mathrm{t}}\right) \frac{\mathrm{ds}}{\mathrm{s}}
\end{aligned}
$$

and from the relation (3.6), it follows

$$
\begin{aligned}
\int_{0}^{+\infty} f_{t} * \phi_{\rho} * \phi_{\rho}(r, x) \frac{d \rho}{\rho} & \frac{1}{t^{2 n+1}} f\left(\frac{r}{t}, \frac{x}{t}\right) \\
= & f_{t}(r, x) .
\end{aligned}
$$

This completes the proof.

Proposition 16 . Let $p, q \in[1,+\infty]$ and $\gamma \in \mathbb{R}$.

The space $\mathscr{B}_{\mathrm{p}, \mathrm{q}}^{\gamma}\left(\left[0,+\infty\left[\times \mathbb{R}^{\mathrm{n}}\right) \cap \mathscr{E}_{*}\left(\mathbb{R} \times \mathbb{R}^{\mathrm{n}}\right)\right.\right.$ is dense in $\mathscr{B}_{\mathrm{p}, \mathrm{q}}^{\gamma}\left(\left[0,+\infty\left[\times \mathbb{R}^{n}\right)\right.\right.$.

Proof. Let $f \in \mathscr{B}_{\mathrm{p}, \mathrm{q}}^{\gamma}\left(\left[0,+\infty\left[\times \mathbb{R}^{n}\right)\right.\right.$ and $\phi \in \mathrm{S}_{*, 0}^{1}\left(\mathbb{R} \times \mathbb{R}^{n}\right)$. For all $\mathrm{t}>0$, the function

$$
(r, x) \longmapsto f * \phi_{t}(r, x)=\left\langle f, \tau_{(r,-x)}\left(\check{\phi_{t}}\right)\right\rangle
$$

belongs to the space $\mathscr{E}_{*}\left(\mathbb{R} \times \mathbb{R}^{n}\right)$ and is slowly increasing. From $i$ ) of Proposition 8 , we deduce that the function $f * \phi_{\mathrm{t}} * \phi_{\mathrm{t}}$ belongs to the space $\mathscr{E}_{*}\left(\mathbb{R} \times \mathbb{R}^{n}\right)$. Thus, from derivative's theorem it follows that for all $k \in \mathbb{N}^{*}$; the function

$$
f_{k}(r, x)=\int_{\frac{1}{k}}^{k} f * \phi_{t} * \phi_{t}(r, x) \frac{d t}{t}
$$

is infinitely differentiable on $\mathbb{R} \times \mathbb{R}^{n}$, even with respect to the first variable. On the other hand, let $\psi \in S_{*, 0}^{1}\left(\mathbb{R} \times \mathbb{R}^{n}\right)$, by Fubini's theorem, we have

$$
f_{k} * \psi_{\rho}=\int_{\frac{1}{k}}^{k} f * \phi_{t} * \phi_{t} * \psi_{\rho} \frac{d t}{t} .
$$

And by the same way as the proof of Theorem 14, we deduce that for all $\rho>0$, the function $f_{k} * \psi_{\rho}$ belongs to $L^{p}\left(d v_{n}\right)$ and that the function

$$
\rho \longmapsto \frac{\left\|f_{k} * \psi_{\rho}\right\|_{p, v_{n}}}{\rho^{\gamma}}
$$


belongs to $L^{\mathrm{q}}\left(\frac{\mathrm{d} \rho}{\rho}\right)$. Again, by Fubini's theorem, for all $\psi \in S_{*, 0}^{1}\left(\mathbb{R} \times \mathbb{R}^{n}\right)$,

$$
\begin{aligned}
\int_{0}^{+\infty} \mathrm{f}_{\mathrm{k}} * \psi_{\rho} * \psi_{\rho} \frac{\mathrm{d} \rho}{\rho} & =\int_{0}^{+\infty}\left(\int_{\frac{1}{\mathrm{k}}}^{\mathrm{k}} \mathrm{f} * \phi_{\mathrm{t}} * \phi_{\mathrm{t}} * \psi_{\rho} * \psi_{\rho} \frac{\mathrm{dt}}{\mathrm{t}}\right) \frac{\mathrm{d} \rho}{\rho} \\
& =\int_{\frac{1}{k}}^{k}\left(\int_{0}^{+\infty} \mathrm{f} * \psi_{\rho} * \psi_{\rho} * \phi_{\mathrm{t}} * \phi_{\mathrm{t}} \frac{\mathrm{d} \rho}{\rho}\right) \frac{\mathrm{dt}}{\mathrm{t}}
\end{aligned}
$$

and by Lemma 13 and Theorem 14, we obtain

$$
\begin{aligned}
\int_{0}^{+\infty} f_{k} * \psi_{\rho} * \psi_{\rho} \frac{d \rho}{\rho} & =\int_{\frac{1}{k}}^{k} f * \phi_{t} * \phi_{t} \frac{d t}{t} \\
& =f_{k} .
\end{aligned}
$$

This shows that for all $k \in \mathbb{N}^{*}$, the function $f_{k}$ belongs to the space $\mathscr{B}_{p, q}^{\gamma}\left(\left[0,+\infty\left[\times \mathbb{R}^{n}\right) \cap \mathscr{E}_{*}\left(\mathbb{R} \times \mathbb{R}^{n}\right)\right.\right.$. Moreover, for every $\varphi \in S_{*, 0}^{1}\left(\mathbb{R} \times \mathbb{R}^{n}\right)$, we have

$$
f_{k} * \varphi_{\rho}=\int_{\frac{1}{k}}^{k} f * \phi_{t} * \phi_{t} * \varphi_{\rho} \frac{d t}{t}
$$

and by i) of Lemma 13, we get

$$
\mathrm{f} * \varphi_{\rho}=\int_{0}^{+\infty} \mathrm{f} * \phi_{\mathrm{t}} * \phi_{\mathrm{t}} * \varphi_{\rho} \frac{\mathrm{dt}}{\mathrm{t}}
$$

Thus,

$$
\begin{aligned}
\left(f-f_{k}\right) * \varphi_{\rho} & =\int_{0}^{\frac{1}{k}} f * \phi_{t} * \phi_{t} * \varphi_{\rho} \frac{d t}{t}+\int_{k}^{+\infty} f * \phi_{t} * \phi_{t} * \varphi_{\rho} \frac{d t}{t} \\
& =\int_{\left[0, \frac{1}{k}\right] \cup[k,+\infty[} f * \phi_{t} * \phi_{t} * \varphi_{\rho} \frac{d t}{t} .
\end{aligned}
$$

Now using the relation (3.13), we obtain

$$
\begin{aligned}
\left(f-f_{k}\right) * \varphi_{\rho} & =\int_{\left([ 0 , \frac { 1 } { k \rho } ] \cup \left[\frac{k}{\rho},+\infty[) \cap[\alpha, \beta]\right.\right.} f * \phi_{\rho s} * \phi_{\rho s} * \varphi_{\rho} \frac{d s}{s} \\
& =\int_{0}^{+\infty} \mathbf{1}_{\left([ 0 , \frac { 1 } { k \rho } ] \cup \left[\frac{k}{\rho},+\infty[) \cap[\alpha, \beta]\right.\right.}(s) f * \phi_{\rho s} * \phi_{\rho s} * \varphi_{\rho} \frac{d s}{s} .
\end{aligned}
$$

Now Minkowski's inequality leads to

$$
\begin{aligned}
\left\|\left(f-f_{k}\right) * \varphi_{\rho}\right\|_{p, v_{n}} & \leqslant \int_{\alpha}^{\beta} 1_{\left([ 0 , \frac { 1 } { k \rho } ] \cup \left[\frac{k}{\rho},+\infty[)\right.\right.}(s)\left\|f * \phi_{\rho s} * \phi_{\rho s} * \varphi_{\rho}\right\|_{p, v_{n}} \frac{d s}{s} \\
& \leqslant \int_{\alpha}^{\beta} 1_{\left([ 0 , \frac { 1 } { k \rho } ] \cup \left[\frac{k}{\rho},+\infty[)\right.\right.}(s)\left\|f * \phi_{\rho s}\right\|_{p, v_{n}}\left\|\phi_{\rho s} * \varphi_{\rho}\right\|_{1, v_{n}} \frac{d s}{s} \\
& \leqslant\|\phi\|_{1, v_{n}}\|\varphi\|_{1, v_{n}} \int_{\alpha}^{\beta} 1_{\left([ 0 , \frac { 1 } { k \rho } ] \cup \left[\frac{k}{\rho},+\infty[)\right.\right.}(s)\left\|f * \phi_{\rho s}\right\|_{p, v_{n}} \frac{d s}{s} .
\end{aligned}
$$


Consequently;

$$
\begin{aligned}
\frac{\left\|\left(f-f_{k}\right) * \varphi_{\rho}\right\|_{p, v_{n}}}{\rho^{\gamma}} & \leqslant\|\phi\|_{1, v_{n}}\|\varphi\|_{1, v_{n}} \int_{\alpha}^{\beta} \mathbf{1}_{\left(\left[0, \frac{1}{k}\right] \cup[k,+\infty[)\right.}(\rho s) \frac{\left\|f * \phi_{\rho s}\right\|_{p, v_{n}}}{\rho^{\gamma}} \frac{d s}{s} \\
& \leqslant\|\phi\|_{1, v_{n}}\|\varphi\|_{1, v_{n}} \int_{\frac{1}{\beta}}^{\frac{1}{\alpha}} \mathbf{1}_{\left(\left[0, \frac{1}{k}\right] \cup[k,+\infty[)\right.}\left(\frac{\rho}{t}\right) t^{-\gamma} \frac{\left\|f * \phi_{\frac{\rho}{t}}\right\|_{p, v_{n}}}{\left(\frac{\rho}{t}\right)^{\gamma}} \frac{d t}{t} \\
& =\|\phi\|_{1, v_{n}}\|\varphi\|_{1, v_{n}}\left(t^{-\gamma} \mathbf{1}_{\left[\frac{1}{\beta}, \frac{1}{\alpha}\right]} \star \frac{\left\|f * \phi_{t}\right\|_{p, v_{n}}}{t^{\gamma}} \mathbf{1}_{\left(\left[0, \frac{1}{k}\right] \cup[k,+\infty[)\right.}\right)(\rho) .
\end{aligned}
$$

Thus, by the relation (3.3), we obtain

$$
\begin{aligned}
M_{\mathcal{p}, \boldsymbol{q}}^{\gamma, \varphi}\left(f_{k}-f\right) \leqslant\|\phi\|_{1, v_{n}} & \|\varphi\|_{1, v_{n}}\left\|t^{-\gamma} \mathbf{1}_{\left[\frac{1}{\beta}, \frac{1}{\alpha}\right]}\right\|_{L^{1}\left(\frac{d t}{t}\right)} \\
& \times\left[\int_{0}^{\frac{1}{k}}\left(\frac{\left\|f * \phi_{t}\right\|_{p, v_{n}}}{t^{\gamma}}\right)^{q} \frac{d t}{t}+\int_{k}^{+\infty}\left(\frac{\left\|f * \phi_{t}\right\|_{p}, v_{n}}{t^{\gamma}}\right)^{q} \frac{d t}{t}\right]^{\frac{1}{q}} .
\end{aligned}
$$

So,

$$
\lim _{k \rightarrow+\infty} M_{p, q}^{\gamma, \varphi}\left(f_{k}-f\right)=0
$$

because

$$
\int_{0}^{+\infty}\left(\frac{\left\|\mathrm{f} * \phi_{\mathrm{t}}\right\|_{p, v_{n}}}{\mathrm{t}^{\gamma}}\right)^{\mathrm{q}} \frac{\mathrm{dt}}{\mathrm{t}}<+\infty
$$

and the proof is complete.

We denote by $L^{\mathrm{q}}(] 0,+\infty\left[, \mathrm{L}^{\mathrm{p}}\left(\mathrm{d} v_{n}\right), \frac{d t}{t}\right)$ the space of measurable functions $g$ on $] 0,+\infty[\times$ $\left[0,+\infty\left[\times \mathbb{R}^{n}\right.\right.$ such that for all $t>0$, the function $g(t,(.,)$.$) belongs to the space L^{p}\left(d v_{n}\right)$ and the function

$$
\mathrm{t} \longmapsto\|\mathrm{g}(\mathrm{t},(., .))\|_{p, v_{n}}
$$

belongs to $L^{\mathrm{q}}\left(\frac{\mathrm{dt}}{\mathrm{t}}\right)$. This space is equipped with the norm

$$
\|g\|_{L^{q}(] 0,+\infty\left[, L^{p}\left(d v_{n}\right), \frac{d t}{t}\right)}=\left(\int_{0}^{+\infty}\|g(t,(., .))\|_{p, v_{n}}^{q} \frac{d t}{t}\right)^{\frac{1}{q}} .
$$

Then we have

Lemma 17 . Let $p, q \in[1,+\infty]$ and let $\gamma<(2 n+1) / p$. For all $\phi \in S_{*, 0}^{1}\left(\mathbb{R} \times \mathbb{R}^{n}\right)$, the mapping $\mathrm{F}$ defined by

$$
F(g)(r, x)=\int_{0}^{+\infty} t^{\gamma} g(t,(., .)) * \phi_{t}(r, x) \frac{d t}{t}
$$

is continuous from $L^{q}(] 0,+\infty\left[, L^{p}\left(d v_{n}\right), \frac{d t}{t}\right)$ into $\mathscr{B}_{p, q}^{\gamma}\left(\left[0,+\infty\left[\times \mathbb{R}^{n}\right)\right.\right.$. 
Proof. Let $\phi \in \mathrm{S}_{*, 0}^{1}\left(\mathbb{R} \times \mathbb{R}^{\mathfrak{n}}\right)$ and $\mathrm{g} \in \mathrm{L}^{\mathrm{q}}(] 0,+\infty\left[, \mathrm{L}^{\mathrm{p}}\left(\mathrm{d} v_{\mathrm{n}}\right), \frac{\mathrm{dt}}{\mathrm{t}}\right)$.

- Let $a, b$ be real numbers such that $b>a>0$ and

$$
\mathscr{F}(\phi)(\mu, \lambda)=0 \text { if } \mu^{2}+2|\lambda|^{2}<a^{2} \quad \text { or } \mu^{2}+2|\lambda|^{2}>b^{2} .
$$

Let $\psi \in S_{*}\left(\mathbb{R} \times \mathbb{R}^{n}\right)$ such that

$$
\mathscr{F}(\psi)(\mu, \lambda)=1 \quad \text { if } \quad a^{2} \leqslant \mu^{2}+2|\lambda|^{2} \leqslant b^{2},
$$

then from the relation (2.9), we deduce that for every $t>0$

$$
\psi_{\mathrm{t}} * \phi_{\mathrm{t}}=\phi_{\mathrm{t}} .
$$

For every $k \in \mathbb{N}^{*}$, the function $F(g)_{k}$ defined by

$$
\mathrm{F}(\mathrm{g})_{\mathrm{k}}(\mathrm{r}, \mathrm{x})=\int_{\frac{1}{\mathrm{k}}}^{\mathrm{k}} \mathrm{t}^{\gamma} \mathrm{g}(\mathrm{t},(., .)) * \phi_{\mathrm{t}}(\mathrm{r}, \mathrm{x}) \frac{\mathrm{dt}}{\mathrm{t}} .
$$

is bounded on $\mathbb{R} \times \mathbb{R}^{n}$. In fact, from the relations (2.7) and (3.4), we deduce that for all $(r, x) \in$ $\mathbb{R} \times \mathbb{R}^{n}$,

$$
\begin{aligned}
\left|F(g)_{k}(r, x)\right| & \leqslant \int_{\frac{1}{k}}^{k} t^{\gamma}\|g(t,(., .))\|_{p, v_{n}}\left\|\phi_{t}\right\|_{p^{\prime}, v_{n}} \frac{d t}{t} \\
& \leqslant\|\phi\|_{p^{\prime}, v_{n}} \int_{\frac{1}{k}}^{k} t^{\gamma-\frac{2 n+1}{p}}\|g(t,(., .))\|_{p, v_{n}} \frac{d t}{t} \\
& \leqslant\|\phi\|_{p^{\prime}, v_{n}}\|g\|_{L^{q}(] 0,+\infty\left[, L^{p}\left(d v_{n}\right), \frac{d t}{t}\right)}\left[\int_{\frac{1}{k}}^{k} t^{\left(\gamma-\frac{2 n+1}{p}\right)} q^{\prime} \frac{d t}{t}\right]^{\frac{1}{q^{\prime}}} \\
& <+\infty .
\end{aligned}
$$

Thus, for all $k \in \mathbb{N}^{*}$ the function $F(g)_{k}$ defines a tempered distribution on $\mathbb{R} \times \mathbb{R}^{n}$, even with respect to the first variable. Moreover, for all $h \in S_{*}\left(\mathbb{R} \times \mathbb{R}^{n}\right)$, we have

$$
\begin{aligned}
\left\langle\mathrm{F}(\mathrm{g})_{\mathrm{k}}, \mathrm{h}\right\rangle & =\int_{\frac{1}{k}}^{\mathrm{k}} \mathrm{t}^{\gamma}\left[\int_{0}^{+\infty} \int_{\mathbb{R}^{n}} h(r, x) g(t,(., .)) * \phi_{\mathrm{t}}(\mathrm{r}, \mathrm{x}) \mathrm{d} v_{\mathrm{n}}(\mathrm{r}, \mathrm{x})\right] \frac{\mathrm{dt}}{\mathrm{t}} \\
& =\int_{\frac{1}{k}}^{\mathrm{k}} \mathrm{t}^{\gamma}\left\langle\mathrm{g}(\mathrm{t},(., .)) * \phi_{\mathrm{t}}, \mathrm{h}\right\rangle \frac{\mathrm{dt}}{\mathrm{t}}
\end{aligned}
$$

and by the relation (3.17), it follows that

$$
\begin{aligned}
\left\langle\mathrm{F}(\mathrm{g})_{\mathrm{k}}, \mathrm{h}\right\rangle & =\int_{\frac{1}{\mathrm{k}}}^{\mathrm{k}} \mathrm{t}^{\gamma}\left\langle\mathrm{g}(\mathrm{t},(., .)) * \phi_{\mathrm{t}} * \psi_{\mathrm{t}}, \mathrm{h}\right\rangle \frac{\mathrm{dt}}{\mathrm{t}} \\
& =\int_{\frac{1}{k}}^{k} \mathrm{t}^{\gamma}\left\langle\mathrm{g}(\mathrm{t},(., .)) * \phi_{\mathrm{t}}, \mathrm{h} * \check{\psi}_{\mathrm{t}}\right\rangle \frac{\mathrm{dt}}{\mathrm{t}} .
\end{aligned}
$$




\section{However,}

$$
\begin{aligned}
\int_{0}^{+\infty} t^{\gamma} \mid\langle g(t,(., .)) & \left.* \phi_{t}, h * \check{\psi}_{t}\right\rangle \mid \frac{d t}{t} \\
& \leqslant \int_{0}^{+\infty} t^{\gamma}\left\|g(t,(., .)) * \phi_{t}\right\|_{\infty, v_{n}}\left\|h * \check{\psi}_{t}\right\|_{1, v_{n}} \frac{d t}{t} \\
& \leqslant \int_{0}^{+\infty} t^{\gamma}\|g(t,(., .))\|_{p, v_{n}}\left\|\phi_{t}\right\|_{p^{\prime}, v_{n}}\left\|h * \check{\psi}_{t}\right\|_{1, v_{n}} \frac{d t}{t} \\
& =\|\phi\|_{p^{\prime}, v_{n}} \int_{0}^{+\infty} t^{\gamma-\frac{2 n+1}{p}}\|g(t,(., .))\|_{p, v_{n}}\left\|h * \check{\psi}_{t}\right\|_{1, v_{n}} \frac{d t}{t} \\
& =\|\phi\|_{p^{\prime}, v_{n}}\left\{\int_{0}^{1} t^{\gamma-\frac{2 n+1}{p}}\|g(t,(., .))\|_{p, v_{n}}\left\|h * \check{\psi}_{t}\right\|_{1, v_{n}} \frac{d t}{t}\right. \\
& \left.+\int_{1}^{+\infty} t^{\gamma-\frac{2 n+1}{p}}\|g(t,(., .))\|_{p, v_{n}}\left\|h * \check{\psi}_{t}\right\|_{1, v_{n}} \frac{d t}{t}\right\}
\end{aligned}
$$

Applying the relations (3.7) and (3.8), we get

$$
\begin{aligned}
& \int_{0}^{+\infty} t^{\gamma}\left|\left\langle g(t,(., .)) * \phi_{t}, h * \check{\psi}_{t}\right\rangle\right| \frac{d t}{t} \\
& \leqslant\|\phi\|_{p^{\prime}, v_{n}}\left\|\Delta^{k} h\right\|_{1, v_{n}}\left\|\check{\psi}_{k}\right\|_{1, v_{n}} \int_{0}^{1} t^{2 k+\gamma-\frac{2 n+1}{p}}\|g(t,(., .))\|_{p, v_{n}} \frac{d t}{t} \\
& +\|\phi\|_{p^{\prime}, v_{n}}\|h\|_{1, v_{n}}\|\check{\psi}\|_{1, v_{n}} \int_{1}^{+\infty} t^{\gamma-\frac{2 n+1}{p}}\|g(t,(., .))\|_{p, v_{n}} \frac{d t}{t} ;
\end{aligned}
$$

and by Hölder's inequality, we have

$$
\begin{aligned}
& \int_{0}^{+\infty} t^{\gamma}\left|\left\langle g(t,(., .)) * \phi_{t}, h * \check{\psi}_{t}\right\rangle\right| \frac{d t}{t} \\
& \leqslant\|\phi\|_{p^{\prime}, v_{n}}\|g\|_{L^{a}\left(10,+\infty\left[, L^{p}\left(d v_{n}\right), \frac{d t}{t}\right)\right.}\left\{\left\|\Delta^{k} h\right\|_{1, v_{n}}\left\|\check{\psi_{k}}\right\|_{1, v_{n}}\left(\int_{0}^{1} t^{\left(2 k+\gamma-\frac{2 n+1}{p}\right) q^{\prime}} \frac{d t}{t}\right)^{\frac{1}{q^{\prime}}}\right. \\
& \left.+\|\mathrm{h}\|_{1, v_{n}}\|\check{\psi}\|_{1, v_{n}}\left(\int_{1}^{+\infty} \mathrm{t}^{\left(\gamma-\frac{2 n+1}{p}\right)} \mathrm{q}^{\prime} \frac{\mathrm{dt}}{\mathrm{t}}\right)^{\frac{1}{q^{\prime}}}\right\}<+\infty .
\end{aligned}
$$

The last inequality together with the relation (3.18) show that for all $h \in S_{*}\left(\mathbb{R} \times \mathbb{R}^{n}\right)$, $\lim _{k \rightarrow+\infty}\left\langle F(g)_{k}, h\right\rangle$ exists and

$$
\lim _{k \rightarrow+\infty}\left\langle F(g)_{k}, h\right\rangle=\int_{0}^{+\infty} t^{\gamma}\left\langle g(t,(., .)) * \phi_{t}, h\right\rangle \frac{d t}{t} .
$$

Consequently, the function

$$
F(g)(r, x)=\int_{0}^{+\infty} t^{\gamma} g(t,(., .)) * \phi_{t}(r, x) \frac{d t}{t}
$$

defines an element of $S_{*}^{\prime}\left(\mathbb{R} \times \mathbb{R}^{\mathfrak{n}}\right)$. 
- Let $\varphi \in S_{*, 0}^{1}\left(\mathbb{R} \times \mathbb{R}^{n}\right)$, we have

$$
\begin{aligned}
\mathrm{F}(\mathrm{g}) * \varphi_{\rho}(\mathrm{r}, \mathrm{x}) & =\left\langle\mathrm{F}(\mathrm{g}), \tau_{(\mathrm{r},-x)} \check{\varphi}_{\rho}\right\rangle \\
& =\lim _{k \rightarrow+\infty}\left\langle\mathrm{F}(\mathrm{g})_{k}, \tau_{(\mathrm{r},-x)} \check{\varphi}_{\rho}\right\rangle \\
& =\lim _{k \rightarrow+\infty} \mathrm{F}(\mathrm{g})_{k} * \varphi_{\rho}(\mathrm{r}, x) \\
& =\lim _{k \rightarrow+\infty} \int_{\frac{1}{k}}^{k} \mathrm{t}^{\gamma} \mathrm{g}(\mathrm{t},(., .)) * \phi_{\mathrm{t}} * \varphi_{\rho}(\mathrm{r}, x) \frac{\mathrm{dt}}{\mathrm{t}} .
\end{aligned}
$$

However, the relation (3.13) implies

$$
\begin{aligned}
& \int_{0}^{+\infty} \mathrm{t}^{\gamma}\left|g(t,(., .)) * \phi_{\mathrm{t}} * \varphi_{\rho}(\mathrm{r}, \mathrm{x})\right| \frac{\mathrm{dt}}{\mathrm{t}} \int_{\rho \alpha}^{\rho \beta} \mathrm{t}^{\gamma}\left|\mathrm{g}(\mathrm{t},(., .)) * \phi_{\mathrm{t}} * \varphi_{\rho}(\mathrm{r}, \mathrm{x})\right| \frac{\mathrm{dt}}{\mathrm{t}} \\
& \leqslant \int_{\rho \alpha}^{\rho \beta} t^{\gamma}\|g(t,(., .))\|_{p, v_{n}}\left\|\phi_{t} * \varphi_{\rho}\right\|_{p^{\prime}, v_{n}} \frac{d t}{t} \\
& \leqslant\|\phi\|_{p^{\prime}, v_{n}}\|\varphi\|_{1, v_{n}} \int_{\rho \alpha}^{\rho \beta} t^{\gamma-\frac{2 n+1}{p}}\|g(t,(., .))\|_{p, v_{n}} \frac{d t}{t}, \\
& \leqslant\|\phi\|_{p^{\prime}, v_{n}}\|\varphi\|_{1, v_{n}}\left(\int_{\rho \alpha}^{\rho \beta} t^{\left(\gamma-\frac{2 n+1}{p}\right)} q^{\prime} \frac{d t}{t}\right)^{\frac{1}{q^{\prime}}}\|g\|_{L^{q}(] 0,+\infty\left[, L^{p}\left(d v_{n}\right), \frac{d t}{t}\right)} \\
& <+\infty \text {. }
\end{aligned}
$$

Thus,

$$
\begin{aligned}
\mathrm{F}(\mathrm{g}) * \varphi_{\rho}(\mathrm{r}, \mathrm{x}) & =\int_{0}^{+\infty} \mathrm{t}^{\gamma} \mathrm{g}(\mathrm{t},(., .)) * \phi_{\mathrm{t}} * \varphi_{\rho}(\mathrm{r}, \mathrm{x}) \frac{\mathrm{dt}}{\mathrm{t}} \\
& =\int_{\alpha}^{\beta}(\rho s)^{\gamma} \mathrm{g}(\rho s,(., .)) * \phi_{\rho s} * \varphi_{\rho}(\mathrm{r}, \mathrm{x}) \frac{\mathrm{ds}}{\mathrm{s}} .
\end{aligned}
$$

By Minkowski's inequality, we obtain

$$
\begin{aligned}
\left\|\mathrm{F}(\mathrm{g}) * \varphi_{\rho}\right\|_{p, v_{n}} & \leqslant \int_{\alpha}^{\beta}(\rho s)^{\gamma}\left\|\mathrm{g}(\rho s,(., .)) * \phi_{\rho s} * \varphi_{\rho}\right\|_{p, v_{n}} \frac{\mathrm{d} s}{\mathrm{~s}} \\
& \leqslant\|\phi\|_{1, v_{n}}\|\varphi\|_{1, v_{n}} \int_{\alpha}^{\beta}(\rho s)^{\gamma}\|\mathrm{g}(\rho s,(., .))\|_{p, v_{n}} \frac{\mathrm{d} s}{s} \\
& <+\infty
\end{aligned}
$$

and

$$
\begin{aligned}
\frac{\left\|\mathrm{F}(\mathrm{g}) * \varphi_{\rho}\right\|_{p, v_{n}}}{\rho^{\gamma}} & \leqslant\|\phi\|_{1, v_{n}}\|\varphi\|_{1, v_{n}} \int_{\alpha}^{\beta} s^{\gamma}\|g(\rho s,(., .))\|_{p, v_{n}} \frac{\mathrm{d} s}{\mathrm{~s}} \\
& =\|\phi\|_{1, v_{n}}\|\varphi\|_{1, v_{n}} \int_{\frac{1}{\beta}}^{\frac{1}{\alpha}} \mathrm{t}^{-\gamma}\left\|\mathrm{g}\left(\frac{\rho}{\mathrm{t}},(., .)\right)\right\|_{p, v_{n}} \frac{\mathrm{dt}}{\mathrm{t}} \\
& =\|\phi\|_{1, v_{n}}\|\varphi\|_{1, v_{n}}\left(\mathrm{t}^{-\gamma} \mathbf{1}_{\left[\frac{1}{\beta}, \frac{1}{\alpha}\right]} \star\|g(t,(., .))\|_{p, v_{n}}\right)(\rho),
\end{aligned}
$$


and by the relation (3.3) it follows that

$$
\begin{aligned}
\left\|\frac{\left\|F(g) * \varphi_{\rho}\right\|_{p, v_{n}}}{\rho^{\gamma}}\right\|_{L^{q}\left(\frac{d \rho}{\rho}\right)} & \leqslant\|\phi\|_{1, v_{n}}\|\varphi\|_{1, v_{n}}\left\|t^{-\gamma} \mathbf{1}_{\left[\frac{1}{\beta}, \frac{1}{\alpha}\right]}\right\|_{L^{1}\left(\frac{d t}{t}\right)}\|g\|_{L^{q}(] 0,+\infty\left[, L^{p}\left(d v_{n}\right), \frac{d t}{t}\right)} \\
& <+\infty .
\end{aligned}
$$

- Let $\varphi \in S_{*, 0}^{1}\left(\mathbb{R} \times \mathbb{R}^{n}\right)$, from the relation (3.19), we have

$$
\mathrm{F}(\mathrm{g}) * \varphi_{\rho}(\mathrm{r}, \mathrm{x})=\int_{\alpha}^{\beta}(\rho s)^{\gamma} \mathrm{g}(\rho s,(., .)) * \phi_{\rho s} * \varphi_{\rho}(\mathrm{r}, \mathrm{x}) \frac{\mathrm{ds}}{\mathrm{s}},
$$

and by Fubini's theorem, we get

$$
\begin{aligned}
\mathrm{F}(\mathrm{g}) * \varphi_{\rho} * \varphi_{\rho}(\mathrm{r}, \mathrm{x}) & =\int_{\alpha}^{\beta}(\rho s)^{\gamma} \mathrm{g}(\rho s,(., .)) * \phi_{\rho s} * \varphi_{\rho} * \varphi_{\rho}(\mathrm{r}, \mathrm{x}) \frac{\mathrm{d} s}{\mathrm{~s}} \\
& =\int_{\rho \alpha}^{\rho \beta} \mathrm{t}^{\gamma} \mathrm{g}(\mathrm{t},(., .)) * \phi_{\mathrm{t}} * \varphi_{\rho} * \varphi_{\rho}(\mathrm{r}, \mathrm{x}) \frac{\mathrm{dt}}{\mathrm{t}} .
\end{aligned}
$$

Thus,

$$
\begin{aligned}
\int_{\frac{1}{k}}^{k} F(g) * \varphi_{\rho} * \varphi_{\rho}(r, x) \frac{d \rho}{\rho} \int_{\frac{1}{k}}^{k} & {\left[\int_{\rho \alpha}^{\rho \beta} t^{\gamma} g(t,(., .)) * \phi_{t} * \varphi_{\rho} * \varphi_{\rho}(r, x) \frac{d t}{t}\right] \frac{d \rho}{\rho} } \\
& =\int_{\frac{\alpha}{k}}^{\beta k} t^{\gamma}\left[\int_{\frac{t}{\beta}}^{\frac{t}{\alpha}} g(t,(., .)) * \phi_{t} * \varphi_{\rho} * \varphi_{\rho}(r, x) \frac{d \rho}{\rho}\right] \frac{d t}{t} .
\end{aligned}
$$

However,

$$
\begin{aligned}
\int_{\frac{t}{\beta}}^{\frac{t}{\alpha}} g(t,(., .)) & * \phi_{t} * \varphi_{\rho} * \varphi_{\rho}(r, x) \frac{d \rho}{\rho} \\
& =\int_{\frac{t}{\beta}}^{\frac{t}{\alpha}}\left[\int_{0}^{+\infty} \int_{\mathbb{R}^{n}} \tau_{(r,-x)} \check{g}(t,(., .))(s, y) \phi_{t} * \varphi_{\rho} * \varphi_{\rho}(s, y) d v_{n}(s, y)\right] \frac{d \rho}{\rho} .
\end{aligned}
$$

Again, by Fubini's theorem, we have

$$
\begin{aligned}
\int_{\frac{t}{\beta}}^{\frac{t}{\alpha}} g(t,(., .)) & * \phi_{t} * \varphi_{\rho} * \varphi_{\rho}(r, x) \frac{d \rho}{\rho} \\
& =\int_{0}^{+\infty} \int_{\mathbb{R}^{n}} \tau_{(r,-x)} \check{g}(t,(., .))(s, y)\left[\int_{0}^{+\infty} \phi_{t} * \varphi_{\rho} * \varphi_{\rho}(s, y) \frac{d \rho}{\rho}\right] d v_{\mathfrak{n}}(s, y) .
\end{aligned}
$$

applying 2) of Lemma 13 we obtain

$$
\begin{aligned}
\int_{\frac{t}{\beta}}^{\frac{t}{\alpha}} g(t,(., .)) * \phi_{t} * \varphi_{\rho} * \varphi_{\rho}(r, x) \frac{d \rho}{\rho} & =\int_{0}^{+\infty} \int_{\mathbb{R}^{n}} \tau_{(r,-x)} \check{g}(t,(., .))(s, y) \phi_{t}(s, y) d v_{n}(s, y) \\
& =g(t,(., .)) * \phi_{t}(r, x) .
\end{aligned}
$$


Replacing in the equality (3.21), it follows that

$$
\int_{\frac{1}{k}}^{k} F(g) * \varphi_{\rho} * \varphi_{\rho}(r, x) \frac{d \rho}{\rho}=\int_{\frac{\alpha}{k}}^{\beta k} t^{\gamma} g(t,(., .)) * \phi_{t}(r, x) \frac{d t}{t} .
$$

Hence,

$$
\int_{0}^{+\infty} \mathrm{F}(\mathrm{g}) * \varphi_{\rho} * \varphi_{\rho} \frac{\mathrm{d} \rho}{\rho}=\mathrm{F}(\mathrm{g})
$$

This shows that the function $\mathrm{F}(\mathrm{g})$ belongs to the space $\mathscr{B}_{\mathrm{p}, \mathrm{q}}^{\gamma}\left(\left[0,+\infty\left[\times \mathbb{R}^{n}\right)\right.\right.$ and from the inequality (3.20), we have

$$
M_{p, q}^{\gamma, \varphi}(F(g)) \leqslant\|\phi\|_{1, v_{n}}\|\varphi\|_{1, v_{n}}\left\|t^{-\gamma} \mathbf{1}_{\left[\frac{1}{\beta}, \frac{1}{\alpha}\right]}\right\|_{L^{1}\left(\frac{d t}{t}\right)}\|g\|_{L^{q}(] 0,+\infty\left[, L^{p}\left(d v_{n}\right), \frac{d t}{t}\right)}
$$

which means that the mapping $F$ is continuous from $L^{q}(] 0,+\infty\left[, L^{p}\left(d v_{n}\right), \frac{d t}{t}\right)$ into $\mathscr{B}_{\mathrm{p}, \mathrm{q}}^{\gamma}\left(\left[0,+\infty\left[\times \mathbb{R}^{\mathrm{n}}\right)\right.\right.$.

Theorem 18. Let $p, q \in[1,+\infty]$ and let $\gamma \in \mathbb{R}, \gamma<(2 n+1) / p$. Then the Besov space $\mathscr{B}_{\mathrm{p}, \mathrm{q}}^{\gamma}([0,+\infty[\times$ $\left.\mathbb{R}^{n}\right)$ is a Banach one.

Proof. Let $\phi \in S_{*, 0}^{1}\left(\mathbb{R} \times \mathbb{R}^{n}\right)$. We define the mapping $G$ on the space $\mathscr{B}_{p, q}^{\gamma}\left(\left[0,+\infty\left[\times \mathbb{R}^{n}\right)\right.\right.$ by setting

$$
G(f)(t,(r, x))=\frac{f * \phi_{t}(r, x)}{t^{\gamma}}
$$

The mapping $G$ is continuous from $\mathscr{B}_{p, q}^{\gamma}\left(\left[0,+\infty\left[\times \mathbb{R}^{n}\right)\right.\right.$ into $L^{\mathrm{q}}(] 0,+\infty\left[, \mathrm{L}^{\mathrm{p}}\left(\mathrm{d} v_{\mathrm{n}}\right)\right.$, $\left.\frac{\mathrm{dt}}{\mathrm{t}}\right)$ and we have

$$
\|G(f)\|_{L^{q}(] 0,+\infty\left[, L^{p}\left(d v_{n}\right), \frac{d t}{t}\right)}=M_{p, q}^{\gamma, \phi}(f) .
$$

Moreover, for all $f \in \mathscr{B}_{\mathrm{p}, \mathrm{q}}^{\gamma}\left(\left[0,+\infty\left[\times \mathbb{R}^{\mathrm{n}}\right)\right.\right.$, we have

$$
\begin{aligned}
\mathrm{F} \circ \mathrm{G}(\mathrm{f})(\mathrm{r}, \mathrm{x}) & =\int_{0}^{+\infty} \mathrm{t}^{\gamma} \mathrm{G}(\mathrm{f})(\mathrm{t},(., .)) * \phi_{\mathrm{t}}(\mathrm{r}, \mathrm{x}) \frac{\mathrm{dt}}{\mathrm{t}} \\
& =\int_{0}^{+\infty} \mathrm{t}^{\gamma} \frac{f * \phi_{\mathrm{t}} * \phi_{\mathrm{t}}(\mathrm{r}, x)}{\mathrm{t}^{\gamma}} \frac{\mathrm{dt}}{\mathrm{t}} \\
& =\int_{0}^{+\infty} \mathrm{f} * \phi_{\mathrm{t}} * \phi_{\mathrm{t}}(\mathrm{r}, \mathrm{x}) \frac{\mathrm{dt}}{\mathrm{t}}
\end{aligned}
$$

and by ii) of Lemma 13, we get

$$
F \circ G(f)=f .
$$

This equality shows that

$$
\mathrm{G}\left(\mathscr { B } _ { \mathrm { p } , \mathrm { q } } ^ { \gamma } \left(\left[0,+\infty\left[\times \mathbb{R}^{\mathrm{n}}\right)\right) \operatorname{ker}\left(\mathrm{G} \circ \mathrm{F}-\mathrm{Id}_{\left(\mathrm{L}^{\mathrm{q}}\left(10,+\infty\left[, \mathrm{L}^{\mathrm{p}}\left(\mathrm{d} v_{\mathrm{n}}\right), \frac{\mathrm{dt}}{\mathrm{t}}\right)\right)\right.}\right) .\right.\right.
$$


In particular, $G\left(\mathscr{B}_{p, q}^{\gamma}\left(\left[0,+\infty\left[\times \mathbb{R}^{n}\right)\right)\right.\right.$ is a closed subspace of $L^{\mathrm{q}}(] 0,+\infty\left[, \mathrm{L}^{\mathrm{p}}\left(\mathrm{d} v_{\mathrm{n}}\right), \frac{\mathrm{dt}}{\mathrm{t}}\right)$.

Let $\left(f_{k}\right)_{k \in \mathbb{N}}$ be a Cauchy sequence in $\mathscr{B}_{p, q}^{\gamma}\left(\left[0,+\infty\left[\times \mathbb{R}^{n}\right)\right.\right.$. From the relation (3.22), the sequence $\left(G\left(f_{k}\right)\right)_{k}$ is a Cauchy's one in $L^{q}(] 0,+\infty\left[, L^{p}\left(d v_{n}\right), \frac{d t}{t}\right)$.

Since $G\left(\mathscr{B}_{p, q}^{\gamma}\left(\left[0,+\infty\left[\times \mathbb{R}^{n}\right)\right)\right.\right.$ is a closed subspace of $L^{q}(] 0,+\infty\left[, L^{p}\left(d v_{n}\right), \frac{d t}{t}\right)$, then there exists a function $f$ in $\mathscr{B}_{p, q}^{\gamma}\left(\left[0,+\infty\left[\times \mathbb{R}^{n}\right)\right.\right.$ such that

$$
\lim _{k \rightarrow+\infty} G\left(f_{k}\right)=G(f) \quad \text { in } \quad L^{q}(] 0,+\infty\left[, L^{p}\left(d v_{n}\right), \frac{d t}{t}\right) .
$$

Again by the relation (3.22),

$$
\lim _{k \rightarrow+\infty} f_{k}=f \quad \text { in } \mathscr{B}_{p, q}^{\gamma}\left(\left[0,+\infty\left[\times \mathbb{R}^{\mathfrak{n}}\right) .\right.\right.
$$

Proposition 19. i) Let $q \in[1,+\infty], p_{1}, p_{2} \in[1,+\infty] ; p_{1}<p_{2}$ and let $\gamma_{1}$, $\gamma_{2} \in \mathbb{R}$ such that

$$
\frac{2 n+1}{p_{1}}-\gamma_{1}=\frac{2 n+1}{p_{2}}-\gamma_{2}
$$

Then

$$
\mathscr{B}_{\mathrm{p}_{1}, \mathrm{q}}^{\gamma_{1}}\left(\left[0,+\infty\left[\times \mathbb{R}^{\mathrm{n}}\right) \hookrightarrow \mathscr{B}_{\mathrm{p}_{2}, \mathrm{q}}^{\gamma_{2}}\left(\left[0,+\infty\left[\times \mathbb{R}^{\mathrm{n}}\right) .\right.\right.\right.\right.
$$

ii) For all $p \in[1,+\infty]$,

$$
\mathscr{B}_{\mathrm{p}, 1}^{0}\left(\left[0,+\infty\left[\times \mathbb{R}^{\mathrm{n}}\right) \hookrightarrow \mathrm{L}^{\mathrm{p}}\left(\mathrm{d} v_{\mathrm{n}}\right) .\right.\right.
$$

Proof. i) Let $p_{1}, p_{2}, \gamma_{1}, \gamma_{2}, q$ be real numbers satisfying the hypothesis. Let $p_{3}$ be an exponent such that

$$
\frac{1}{p_{1}}+\frac{1}{p_{3}}=1+\frac{1}{p_{2}} .
$$

Finally, let $f \in \mathscr{B}_{\mathrm{p}_{1}, \mathfrak{q}}^{\gamma}\left(\left[0,+\infty\left[\times \mathbb{R}^{\mathrm{n}}\right)\right.\right.$ and $\phi \in \mathrm{S}_{*, 0}^{1}\left(\mathbb{R} \times \mathbb{R}^{\mathrm{n}}\right)$ such that

$$
\mathscr{F}(\phi)(\mu, \lambda)=0 \text { if } \mu^{2}+2|\lambda|^{2}>b^{2} \text { or } \mu^{2}+2|\lambda|^{2}<a^{2} .
$$

Let us take $\psi \in S_{*}\left(\mathbb{R} \times \mathbb{R}^{n}\right)$ satisfying

$$
\forall(\mu, \lambda) \in \Gamma ; \quad a^{2} \leqslant \mu^{2}+2|\lambda|^{2} \leqslant b^{2}, \quad \mathscr{F}(\psi)(\mu, \lambda)=1 .
$$

Then for all $t>0$, we have

$$
\phi_{\mathrm{t}} * \psi_{\mathrm{t}}=\phi_{\mathrm{t}}
$$

and

$$
\begin{aligned}
M_{p_{2}, q}^{\gamma_{2}, \phi}(f) & =\left(\int_{0}^{+\infty}\left(\frac{\left\|f * \phi_{t}\right\|_{p_{2}, v_{n}}}{t^{\gamma_{2}}}\right)^{\mathrm{q}} \frac{\mathrm{dt}}{\mathrm{t}}\right)^{\frac{1}{q}} \\
& =\left(\int_{0}^{+\infty}\left(\frac{\left\|\mathrm{f} * \phi_{\mathrm{t}} * \psi_{\mathrm{t}}\right\|_{\mathrm{p}_{2}, v_{\mathrm{n}}}}{\mathrm{t}^{\gamma_{2}}}\right)^{\mathrm{q}} \frac{\mathrm{dt}}{\mathrm{t}}\right)^{\frac{1}{q}} .
\end{aligned}
$$


By the relations (2.7), (3.4), (3.23) and (3.24) we get

$$
\begin{aligned}
M_{\mathfrak{p}_{2}, \mathbf{q}}^{\gamma_{2}, \phi}(f) & \leqslant\|\psi\|_{\mathfrak{p}_{3}, v_{n}}\left[\int_{0}^{+\infty}\left(\frac{\left\|f * \phi_{t}\right\|_{p_{1}, v_{n}}}{t^{\gamma_{1}}}\right)^{q} \frac{d t}{t}\right]^{\frac{1}{q}} \\
& \leqslant\|\psi\|_{\mathfrak{p}_{3}, \nu_{n}} M_{\mathfrak{p}_{1}, \mathbf{q}}^{\gamma_{1}, \boldsymbol{f}}(f) .
\end{aligned}
$$

This shows that the space $\mathscr{B}_{p_{1}, \mathfrak{q}}^{\gamma_{1}}\left(\left[0,+\infty\left[\times \mathbb{R}^{n}\right)\right.\right.$ is contained in $\mathscr{B}_{p_{2}, \mathfrak{q}}^{\gamma_{2}}\left(\left[0,+\infty\left[\times \mathbb{R}^{n}\right)\right.\right.$ and that the canonical injection is continuous from $\mathscr{B}_{\mathrm{p}_{1}, \mathrm{q}}^{\gamma_{1}}\left(\left[0,+\infty\left[\times \mathbb{R}^{\mathrm{n}}\right)\right.\right.$ into the space $\mathscr{B}_{\mathrm{p}_{2}, \mathrm{q}}^{\gamma_{2}}\left(\left[0,+\infty\left[\times \mathbb{R}^{\mathrm{n}}\right)\right.\right.$.

ii) Let $f \in \mathscr{B}_{\mathfrak{p}, 1}^{0}\left(\left[0,+\infty\left[\times \mathbb{R}^{\mathfrak{n}}\right) ; p \in[1,+\infty]\right.\right.$. From ii) of Lemma [13, we have

$$
\mathrm{f}=\int_{0}^{+\infty} \mathrm{f} * \phi_{\mathrm{t}} * \phi_{\mathrm{t}} \frac{\mathrm{dt}}{\mathrm{t}} ; \quad \phi \in \mathrm{S}_{*, 0}^{1}\left(\mathbb{R} \times \mathbb{R}^{\mathrm{n}}\right)
$$

thus,

$$
\begin{aligned}
\|f\|_{p, v_{n}} & \leqslant \int_{0}^{+\infty}\left\|f * \phi_{t} * \phi_{t}\right\|_{p, v_{n}} \frac{d t}{t} \\
& \leqslant\|\phi\|_{1, v_{n}} M_{p, 1}^{\mathcal{O}, \phi}(f) .
\end{aligned}
$$

This completes the proof.

In the following, we shall define a discrete norm on the Besov space $\mathscr{B}_{p, q}^{\gamma}\left(\left[0,+\infty\left[\times \mathbb{R}^{n}\right)\right.\right.$ and we will prove that it is equivalent to the norm $M_{\mathcal{p}, q}^{\gamma, \phi} ; \phi \in S_{*, 0}^{1}\left(\mathbb{R} \times \mathbb{R}^{n}\right)$. More precisely, we have Theorem 20. Let $p, q \in[1,+\infty], \gamma \in \mathbb{R}$. Let $a, b$ be real numbers such that $0<\mathrm{a}<\mathrm{b}$ and $\phi \in \mathrm{S}_{*, 0}\left(\mathbb{R} \times \mathbb{R}^{\mathrm{n}}\right)$ verifying

$$
\mathscr{F}(\phi)(\mu, \lambda)=1 \quad \text { if } \quad a^{2} \leqslant \mu^{2}+2|\lambda|^{2} \leqslant b^{2} .
$$

Then the mapping $\mathbf{N}_{\mathbf{p}}^{\gamma, \mathbf{q}}$ defined by

$$
N_{p, q}^{\gamma, \phi}(f)= \begin{cases}\left(\sum_{k \in \mathbb{Z}}\left(\frac{\left\|f * \phi_{2^{k}}\right\|_{p, v_{n}}}{2^{k \gamma}}\right)^{q}\right)^{\frac{1}{q}}, & \text { if } 1 \leqslant q<+\infty ; \\ \underset{k \in \mathbb{Z}}{\operatorname{esssup}} \frac{\left\|f * \phi_{2^{k}}\right\|_{p, v_{n}}}{2^{k \gamma}}, & \text { if } q=+\infty\end{cases}
$$

is a norm on the Besov space $\mathscr{B}_{p, q}^{\gamma}\left(\left[0,+\infty\left[\times \mathbb{R}^{n}\right)\right.\right.$ which defines the same topology as the norm $M_{p, q}^{\gamma, \psi} ; \psi \in S_{*, 0}^{1}\left(\mathbb{R} \times \mathbb{R}^{n}\right)$.

Proof. • From Lemma 9, there exists $\psi \in S_{*, 0}^{1}\left(\mathbb{R} \times \mathbb{R}^{n}\right)$ such that

$$
\mathscr{F}(\psi)(\mu, \lambda)=0 \quad \text { if } \quad \mu^{2}+2|\lambda|^{2}<a^{2} \quad \text { or } \quad \mu^{2}+2|\lambda|^{2}>b^{2} .
$$


Then for all $s \in[1,2]$ and $k \in \mathbb{Z}$, we have

$$
\mathscr{F}(\psi)\left(2^{k} s \mu, 2^{k} s \lambda\right)=\mathscr{F}(\psi)\left(2^{k} s \mu, 2^{k} s \lambda\right) \mathscr{F}(\phi)\left(2^{k} \mu, 2^{k} \lambda\right)
$$

which leads to

$$
\psi_{2^{k} s}=\psi_{2^{k} s} * \phi_{2^{k}}
$$

and therefore, for all $f \in \mathscr{B}_{\mathbf{p}, \mathbf{q}}^{\gamma}\left(\left[0,+\infty\left[\times \mathbb{R}^{n}\right)\right.\right.$

$$
\mathrm{f} * \psi_{2^{\mathrm{k} s}}=\mathrm{f} * \phi_{2^{\mathrm{k}}} * \psi_{2^{\mathrm{k}} \mathrm{s}} .
$$

Then for all $\mathrm{q} \in[1,+\infty[$

$$
\begin{aligned}
M_{p, q}^{\gamma, \Psi}(f) & =\left(\int_{0}^{+\infty}\left(\frac{\left\|f * \psi_{t}\right\|_{p, v_{n}}}{t^{\gamma}}\right)^{q} \frac{d t}{t}\right)^{\frac{1}{q}} \\
& =\left(\sum_{k \in \mathbb{Z}} \int_{2^{k}}^{2^{k+1}}\left(\frac{\left\|f * \psi_{t}\right\|_{p, v_{n}}}{t^{\gamma}}\right)^{q} \frac{d t}{t}\right)^{\frac{1}{q}} \\
& =\left(\sum_{k \in \mathbb{Z}} \int_{1}^{2}\left(\frac{\left\|f * \psi_{2^{k} s}\right\|_{p, v_{n}}}{\left(2^{k} s\right)^{\gamma}}\right)^{q} \frac{d s}{s}\right)^{\frac{1}{q}} .
\end{aligned}
$$

Using the relations (2.7), (3.4) and (3.25), we obtain

$$
\begin{aligned}
M_{p, q}^{\gamma, \Psi}(f) & \leqslant\|\psi\|_{1, v_{n}}\left[\sum_{k \in \mathbb{Z}}\left(\frac{\left\|f * \phi_{2^{k}}\right\|_{p, v_{n}}}{2^{k \gamma}}\right)^{q} \int_{1}^{2} \frac{d s}{s^{\gamma q+1}}\right]^{\frac{1}{q}} \\
& =\|\psi\|_{1, v_{n}}\left(\frac{1-2^{-q \gamma}}{q \gamma}\right)^{\frac{1}{q}} N_{p, q}^{\gamma, \phi}(f) .
\end{aligned}
$$

On the other hand, for $\mathrm{q}=+\infty$ and again by the relation (3.25), we deduce that for all $k \in \mathbb{Z}$ and $s \in[1,2]$

$$
\frac{\left\|f * \psi_{2^{k} s}\right\|_{p, v_{n}}}{\left(2^{k} s\right)^{\gamma}} \leqslant\left(1+2^{-\gamma}\right)\|\psi\|_{1, v_{n}} \frac{\left\|f * \phi_{2^{k}}\right\|_{p, v_{n}}}{2^{k \gamma}} .
$$

Consequently, for all $k \in \mathbb{Z}$ and $t \in\left[2^{k}, 2^{k+1}\right]$

$$
\frac{\left\|\mathrm{f} * \psi_{t}\right\|_{p, v_{n}}}{t^{\gamma}} \leqslant\left(1+2^{-\gamma}\right)\|\psi\|_{1, v_{n}} \mathrm{~N}_{p, \infty}^{\gamma, \phi}(\mathrm{f}),
$$

which shows that

$$
M_{\mathfrak{p}, \infty}^{\gamma, \psi}(f) \leqslant\left(1+2^{-\gamma}\right)\|\psi\|_{1, v_{n}} N_{p}^{\gamma, \phi}(f) .
$$

- Let $\mathrm{a}_{1}, \mathrm{~b}_{1}$ be two real numbers; $0<\mathrm{a}_{1}<\mathrm{a}<\mathrm{b}<\mathrm{b}_{1}$ such that

$$
\mathscr{F}(\phi)(\mu, \lambda)=0 \quad \text { if } \quad \mu^{2}+2|\lambda|^{2}<a_{1}^{2} \quad \text { or } \quad \mu^{2}+2|\lambda|^{2}>b_{1}^{2} .
$$

From Lemma 9, there exists $\psi \in S_{*, 0}^{1}\left(\mathbb{R} \times \mathbb{R}^{n}\right)$ such that

$$
\mathscr{F}(\psi)(\mu, \lambda)=C, \quad \text { for all } \quad(\mu, \lambda) \in \Gamma ; \quad a_{1}^{2} \leqslant \mu^{2}+2|\lambda|^{2} \leqslant 4 b_{1}^{2}
$$


where $C$ is a positive constant. Then for all $k \in \mathbb{Z}$ and $s \in[1,2]$,

$$
\mathrm{C} \mathscr{F}(\phi)\left(2^{k} \mu, 2^{k} \lambda\right)=\mathscr{F}(\phi)\left(2^{k} \mu, 2^{k} \lambda\right) \mathscr{F}(\psi)\left(2^{k} \mu s, 2^{k} \lambda s\right)
$$

so,

$$
\text { C. } \phi_{2^{k}}=\phi_{2^{k}} * \psi_{2^{k} s} .
$$

Hence, for all $f \in \mathscr{B}_{\mathfrak{p}, \mathfrak{q}}^{\gamma}\left(\left[0,+\infty\left[\times \mathbb{R}^{n}\right)\right.\right.$

$$
\mathrm{C} f * \phi_{2^{\mathrm{k}}}=\mathrm{f} * \psi_{2^{\mathrm{k}} \mathrm{s}} * \phi_{2^{\mathrm{k}}}
$$

and

$$
C \frac{\left\|\mathrm{f} * \phi_{2^{k}}\right\|_{p, v_{n}}}{2^{k \gamma}} \leqslant\left(1+2^{\gamma}\right)\|\phi\|_{1, v_{n}} \frac{\left\|\mathrm{f} * \psi_{2^{k} s}\right\|_{p, v_{n}}}{\left(2^{k} s\right)^{\gamma}} .
$$

Integrating over $[1,2]$ with respect to the measure $\frac{d s}{s}$, we get for all $q \in[1,+\infty[$,

$$
\left(\frac{\left\|f * \phi_{2^{k}}\right\|_{p, v_{n}}}{2^{k \gamma}}\right)^{\mathrm{q}} \leqslant \frac{\left(\left(1+2^{\gamma}\right)\|\phi\|_{1, v_{n}}\right)^{\mathrm{q}}}{\mathrm{C}^{\mathrm{q}} \log 2} \int_{2^{k}}^{2^{k+1}}\left(\frac{\left\|\mathrm{f} * \psi_{\mathrm{t}}\right\|_{p, v_{n}}}{\mathrm{t}^{\gamma}}\right)^{\mathrm{q}} \frac{\mathrm{dt}}{\mathrm{t}}
$$

which leads to

$$
\mathrm{N}_{\mathbf{p}, \mathbf{q}}^{\gamma, \phi}(\mathrm{f}) \leqslant \frac{1}{\mathrm{C}}(\log 2)^{-\frac{1}{q}}\left(1+2^{\gamma}\right)\|\phi\|_{1, v_{n}} M_{\mathbf{p}, \mathbf{q}}^{\gamma, \Psi}(f) .
$$

On the other hand, for $\mathrm{q}=+\infty$ and using the relation (3.26), we deduce that for all $k \in \mathbb{Z}$

$$
\frac{\left\|f * \phi_{2^{k}}\right\|_{p, v_{n}}}{2^{k \gamma}} \leqslant \frac{\left(1+2^{\gamma}\right)}{C}\|\phi\|_{1, v_{n}} M_{p, \infty}^{\gamma, \Psi}(f),
$$

which implies that

$$
\mathrm{N}_{p, \infty}^{\gamma, \phi}(f) \leqslant \frac{\left(1+2^{\gamma}\right)}{C}\|\phi\|_{1, v_{n}} M_{p, \infty}^{\gamma, \psi}(f)
$$

This completes the proof of theorem.

Remark 21. 1) From Theorem 14 and Theorem 20, we deduce that the Besov space $\mathscr{B}_{\mathrm{p}, \mathrm{q}}^{\gamma}([0,+\infty[\times$ $\left.\mathbb{R}^{n}\right)$ is independent of the choice of the function $\phi \in S_{*, 0}\left(\mathbb{R} \times \mathbb{R}^{n}\right)$, when it is endowed with the $\operatorname{norm} \mathrm{N}_{\mathbf{p}, \mathbf{q}}^{\gamma, \phi}$.

From Proposition [15] and Theorem 20, we deduce the following proposition

Proposition 22. The Besov space $\mathscr{B}_{p, q}^{\gamma}\left(\left[0,+\infty\left[\times \mathbb{R}^{n}\right)\right.\right.$ is homogeneous in a weaker sense when equipped with the norm $\mathrm{N}_{\mathfrak{p}, q}^{\gamma, q}$, that is there exist $\mathrm{C}_{1}, \mathrm{C}_{2}>0$ such that for all $f \in \mathscr{B}_{\mathfrak{p}, \mathfrak{q}}^{\gamma}([0,+\infty[\times$ $\left.\mathbb{R}^{n}\right)$ and $t>0$

$$
C_{1} t^{\frac{2 n+1}{p}-2 n-1-\gamma} N_{\mathfrak{p}, q}^{\gamma, \phi}(f) \leqslant N_{\mathfrak{p}, q}^{\gamma, \phi}\left(f_{t}\right) \leqslant C_{2} t^{\frac{2 n+1}{p}-2 n-1-\gamma} N_{\mathfrak{p}, q}^{\gamma, \phi}(f) .
$$

Proposition 23. Let $p \in[1,+\infty]$ and $\gamma \in \mathbb{R}$. Then for all $\mathrm{q}_{1}, \mathrm{q}_{2} \in[1,+\infty]$;

$\mathrm{q}_{1} \leqslant \mathrm{q}_{2}$, we have the continuous embedding

$$
\mathscr{B}_{\mathrm{p}, \mathrm{q}_{1}}^{\gamma}\left(\left[0,+\infty\left[\times \mathbb{R}^{\mathrm{n}}\right) \hookrightarrow \mathscr{B}_{\mathrm{p}, \mathrm{q}_{2}}^{\gamma}\left(\left[0,+\infty\left[\times \mathbb{R}^{\mathrm{n}}\right) .\right.\right.\right.\right.
$$


Proof. Let $f \in \mathscr{B}_{p, \mathbf{q}_{1}}^{\gamma}\left(\left[0,+\infty\left[\times \mathbb{R}^{n}\right)\right.\right.$ and $\phi \in S_{*, 0}\left(\mathbb{R} \times \mathbb{R}^{n}\right)$. Since

$$
\sum_{k \in \mathbb{Z}}\left(\frac{\left\|f * \phi_{2^{k}}\right\|_{p, v_{n}}}{2^{k \gamma}}\right)^{q_{1}}<+\infty
$$

then,

$$
\mathrm{N}_{\mathfrak{p}, \infty}^{\gamma, \phi}(\mathrm{f})<+\infty
$$

and we have

$$
\mathrm{N}_{p, \mathbf{q}_{2}}^{\gamma, \phi}(\mathrm{f}) \leqslant\left(\mathrm{N}_{p, \infty}^{\gamma, \phi}(f)\right)^{1-\frac{\mathrm{q}_{1}}{q_{2}}}\left(\mathrm{~N}_{p, \mathbf{q}_{1}}^{\gamma, \phi}(f)\right)^{\frac{\mathrm{q}_{1}}{q_{2}}}
$$

However,

$$
\mathrm{N}_{\mathbf{p}, \infty}^{\gamma, \phi}(f) \leqslant \mathrm{N}_{\mathbf{p}, \mathbf{q}_{1}}^{\gamma, \phi}(f)
$$

and consequently,

$$
\mathbf{N}_{\mathbf{p}, \mathbf{q}_{2}}^{\gamma, \boldsymbol{q}}(f) \leqslant N_{\mathbf{p}, \mathbf{q}_{1}}^{\gamma, \phi}(f)
$$

Received: October 2009. Revised: December 2009.

\section{References}

[1] L. E. Andersson, On the determination of a function from spherical averages, SIAM J. Math. Anal 19 (1988), 214-234.

[2] C. BACCAR, N. B. HAmadi, and L. T. RACHDI, Inversion formulas for the Riemann-Liouville transform and its dual associated with singular partial differential operators. Int. J. Math. Math. Sci. 2006, Article ID 86238, (2006), 1-26.

[3] C. BACCAR, S. OMri, and L. T. RACHDI, Fock spaces connected with spherical mean operator and associated operators. Mediterr. J. Math. 6 (2009), 1-25.

[4] J. J. Betancor and L. Rodriguez-Mesa, On the Besov-Hankel spaces, J. Math. Soc. Japan 50, (3), (1998), 781-788.

[5] O. V. Besov, On a family of function spaces in connection with embeddings and extensions, Tr. Mat. Inst. Steklova 60, (1961), 42-81.

[6] M. Dziri, M. Jelassi and L. T. RAChDI, Spaces of $\mathrm{D}_{\mathrm{L}^{p}}$ type and a convolution product associated with the spherical mean operator. Int. J. Math. Math. Sci.,3,(2005), 357-381.

[7] A. ERdely, Higher Transcendental Functions, vol. II, Mc Graw-Hill, New York, (1953).

[8] A. ERdely, Tables of Integral Transforms, vol. I. Mc GRAW-Hill Book Compagny, INC, (1954). 
[9] J. A. FAwCETT, Inversion of $N$-dimensional spherical means. SIAM J. Appl. Math. 45, (1985), 336-341.

[10] T. M. FletT, Lipschitz spaces of functions on the circle and the disc, J. Math. Anal. Appl. 39 (1972), 125-158.

[11] T. M. Flett, Temperatures, Bessel potentials and Lipschizt spaces, Proc. London Math. Soc. $20,(1970), 749-768$.

[12] G. B. Folland, Real analysis: Modern Techniques and Their Applications. New York, (1984).

[13] D. V. Giang and F. Móriez, A New characterization of Besov spaces on the real line, J. Math. Anal. Appl., 189,(1995), 533-551.

[14] H. Helesten and L. E. Anderson, An inverse method for the processing of synthetic aperture radar data. Inv. Prob. 3 (1987), 111-124.

[15] M. Herberthson, A numerical implementation of an inverse formula for CARABAS Raw Data. Internal Report D 30430-3.2, National Defense Research Institute, Linköping, Sweden, (1986).

[16] L. Kamoun, Besov-type spaces for the Dunkl operator on the real line. J. Comput. Appl. Math. 199, no. 1, (2007), 56-67.

[17] N. N. Lebedev, Special Functions and Their Applications. Dover publications, Inc. NewYork, (1972).

[18] M. M. Nessibi, L. T. Rachdi, and K. Trimèche, Ranges and inversion formulas for spherical mean operator and its dual. J. Math. Anal. Appl. 196, (1995), 861-884.

[19] M. M. Nessibi, and K. Trimèche, Inversion of the Radon transform on the Laguerre hypergroup by using generalized wavelets. J. Math. Anal. Appl. 208 (1997), 337-363.

[20] J. Peetre, New Thoughts on Besov Spaces, Duke Univ. Math. Series, Durham, NC, (1976).

[21] L. T. RACHDI, and K. Trimèche, Weyl transforms associated with the spherical mean operator. Anal. Appl. 1, no 2, (2003), 141-164.

[22] M. Taibleson, On the theory of lipschizt spaces of distributions on euclidean n-space. I, II, III, J. Math. Mech. 13 (1964), 407-480; 65, 821-840; 15 (1966), 973-981.

[23] H. Triebel, Theory of Function Spaces, Monographs in Math., vol. 78, Birkhäuser, Verlag, Basel, (1983).

[24] K. TRIMÈCHE, Transformation intégrale de Weyl et théorème de Paley-Wiener associés a un opérateur différentiel singulier sur $(0,+\infty)$, J. Math. Pures Appl., 60 (1981), 51-98. 
[25] K. Trimèche, Inversion of the Lions translation operator using generalized wavelets, Appl. Comput. Harmon. Anal. 4 (1997), 97-112.

[26] K. Trimèche, Generalized Harmonic Analysis and Wavelet Packets: An Elementary Treatment of Theory \& Applications, Gordon and Breach Science Publishers, (2001).

[27] G. N. Watson, A Treatise on the Theory of Bessel Functions, 2nd ed. Cambridge Univ. Press. London/New-York, (1966). 